ISSN 1855-3966 (printed edn.), ISSN 1855-3974 (electronic edn.)

ARS MATHEMATICA CONTEMPORANEA 17 (2019) 1-35

https://doi.org/10.26493/1855-3974.1800.40c

(Also available at http://amc-journal.eu)

\title{
New methods for finding minimum genus embeddings of graphs on orientable and non-orientable surfaces*
}

\author{
Marston Conder \\ Department of Mathematics, University of Auckland, \\ Private Bag 92019, Auckland 1142, New Zealand
}

Klara Stokes

National University of Ireland Maynooth, Maynooth, Co. Kildare, Ireland and University of Skövde, Sweden

Received 14 September 2018, accepted 4 December 2018, published online 19 June 2019

\begin{abstract}
The question of how to find the smallest genus of all embeddings of a given finite connected graph on an orientable (or non-orientable) surface has a long and interesting history. In this paper we introduce four new approaches to help answer this question, in both the orientable and non-orientable cases. One approach involves taking orbits of subgroups of the automorphism group on cycles of particular lengths in the graph as candidates for subsets of the faces of an embedding. Another uses properties of an auxiliary graph defined in terms of compatibility of these cycles. We also present two methods that make use of integer linear programming, to help determine bounds for the minimum genus, and to find minimum genus embeddings. This work was motivated by the problem of finding the minimum genus of the Hoffman-Singleton graph, and succeeded not only in solving that problem but also in answering several other open questions.
\end{abstract}

Keywords: Graph embedding, genus.

Math. Subj. Class.: 05C10, 05E18, 20B25, 57M15

* The authors are very grateful to Tomaž Pisanski for suggesting that they extend their initial development of the orbit method on the Hoffman-Singleton graph to other graphs, which then led to them to develop the other methods presented here, in order to find the answers to many open questions. The authors are also grateful to the referee for some helpful suggestions about presenting their work. The first author is grateful to the N.Z. Marsden Fund for its support (grant UOA 1626), and acknowledges the use of the MAGMA system [2] for computational experiments and verification of a number of discoveries announced in this paper, as well as Sage [45] in combination with IBM CPLEX for a small number of the ILP computations. The second author acknowledges partial support from the Spanish MEC project ICWT (TIN2016-80250-R) and ARES (CONSOLIDER INGENIO 2010 CSD2007-00004).

E-mail addresses: m.conder@auckland.ac.nz (Marston Conder), klara.stokes@mu.ie (Klara Stokes) 


\section{Introduction}

The question of how to find the smallest genus of those embeddings of a given finite connected graph on an orientable (or non-orientable) surface is a natural extension of determining whether or not a graph is planar, and has a long and interesting history. It is also quite an important question, with applications found in map colouring, topology, finite geometry (configurations and block designs), group theory, number theory and the design of electronic circuits.

Pioneering work was done by Dyck and Heffter in the late 1800s [13, 22], but it was not until the mid-1900s that significant progress was made, leading to the determination by Ringel $[38,39]$ of the minimum non-orientable genus of the complete graph $K_{n}$ (for $n>7$ ) and the minimum orientable and non-orientable genera of each of the complete bipartite graphs $K_{m, n}$, and then the determination by Ringel and Youngs [40] of the minimum orientable genus of the complete graph $K_{n}$ (as a key step towards their proof of the Heawood Map Colouring Problem).

Youngs also gave the first proof of the (now) well known fact that every orientable embedding of a connected graph is determined by the rotations of edges at its vertices [52], and this was taken further by Duke [12] to show that the range of genera of embeddings of a given connected finite graph is an unbroken sequence of non-negative integers (from the minimum genus to the maximum genus of the graph). Similar theory was developed by various people for embeddings on non-orientable surfaces; details may be found in [44]. It is worth noting here that a minimum genus non-orientable embedding of a graph is not necessary a 2-cell embedding, but unless the graph is a tree, there is always at least one minimum genus non-orientable embedding which is a 2-cell embedding; see [35].

In the later 1990s, the minimum orientable genus was found for several graphs and families of graphs, some of which are given in [44, Tables I and II]. In many of these families, the graphs have a large degree of symmetry, which can be helpful to a large extent in finding nice embeddings. Various authors developed a range of techniques that can work well for many classes of graphs, involving rotation systems, voltage graphs, edge insertions and deletions, graph contractions, graph amalgamations and graph products. Some of these are described nicely in Gross and Tucker's book on topological graph theory [19].

On the other hand, some other examples proved quite challenging, even when they were vertex-transitive. Notable cases include the Cartesian product $C_{3} \square C_{3} \square C_{3}$, a 6 valent graph of order 27 which took some years to deal with (see [32, 4]), the 3 -valent Gray graph of order 54 (see [30]), and the associated Doyle-Holt graph, a 4-valent graph of order 27 (considered 13 years ago in [30] and dealt with at last in this paper).

The difficulty is not surprising, even for small graphs, in that a $k$-valent regular graph of order $n$ has $((k-1) !)^{n}$ distinct embeddings into an orientable surface. Furthermore, in 1989 it was shown by Thomassen [47] that the problem of finding the minimum orientable genus of a graph is NP-hard, and the problem of determining whether or not the minimum orientable genus of a connected graph is a given non-negative integer $g$ is NP-complete.

Also the problem of deciding whether or not a graph can be embedded in an orientable surface of given genus $g$ has been considered. A polynomial-time algorithm to solve this problem was presented in 1979 by Filotti, Miller and Reif [14], but then shown in 2011 to be flawed, by Myrvold and Kocay [34]. In the meantime, in 1999 Mohar [31] produced an algorithm for this that runs in linear time in the graph order, but doubly-exponential in the genus. In the case where the graph has no such embedding, the latter algorithm returns a minimal subgraph that cannot be embedded in the given surface, and its validity gives 
a constructive proof of the theorem of Robertson and Seymour [42] for any given closed surface, there are only finitely many minimal forbidden subgraphs.

In contrast, finding the maximum genus of orientable embeddings of graphs is much easier, thanks largely to some work in the 1970s by Xuong, who in [51] gave a formula for this number in terms of the minimum 'deficiency' of spanning trees for the graph. Ten years later Škoviera and Nedela used Xuong's work in [43] to prove that almost every vertex-transitive connected graph is upper-embeddable (in the sense of having a maximum genus embedding with just one or two faces), and indeed that this happens whenever the graph has valency or girth greater than 3 .

In this paper we make further progress on the problem of finding the minimum genus of graphs (in both the orientable and non-orientable cases). Our work was motivated by a question by the second author about the minimum genus of the Hoffman-Singleton graph, which arose in joint work with Izquierdo on geometries associated with Moore graphs [46].

The Hoffman-Singleton graph is the unique Moore graph of valency 7 and diameter 2 (and indeed the largest known Moore graph of diameter 2), and accordingly, is a 7-valent connected graph of order 50 , diameter 2 and girth 5 . The properties of this graph, including its order and valency, made it challenging to find the minimum genus using existing methods (as summarised in [50] for example), and so we had to take a new approach. By considering the action of subgroups of the automorphism group of the graph on cycles of small length, we were able to find a minimum genus embedding on a non-orientable surface with pentagonal faces, and then adapt our approach to find a minimum genus orientable embedding as well.

We wrote up an early version of this paper describing our approach and the results, but perplexingly, had difficulty in getting it accepted by a good journal (despite finding a solution to a very challenging problem and developing a significant new approach in order to do that). Then we got some highly astute advice from Tomaž Pisanski, who suggested that we should apply our new approach to more examples, to underline its effectiveness. So we proceeded to do that, and used our new approach to find (for the first time) the minimum orientable or non-orientable genus of several other graphs, and answer a number of open questions about some of these.

The approach we took for the Hoffman-Singleton graph, which we call the subgroup orbit method, is useful for finding embeddings of graphs on surfaces with a certain degree of symmetry. The method considers candidates for a subgroup $G$ of suitable order in the automorphism group of the graph such that $G$ induces a group of automorphisms of the embedding, and this helps to reduce the complexity of the search for such an embedding. The automorphism group of a graph embedding is a subgroup of the automorphism group of the underlying graph, and acts semi-regularly on the 'flags' of the embedding (see Subsection 3.1), so $|G|$ must divide the number of flags, which is four times the number of edges of the graph. Orbits of $G$ on closed walks of chosen lengths in the graph are taken as possibilities for the boundaries of faces of the embedding, and then tested for compatibility, completeness and orientability.

The subgroup orbit method works well for finding embeddings with face-transitive automorphism group, but can also work well in other cases where the automorphism group of the embedding has a small number of orbits on faces, and the lengths of those faces are close to the girth of the graph. But of course it is a lot to expect such properties, and indeed for some of the graphs we investigated, there were no such embeddings. For those, we had to develop other methods, which appear to be new as well. 
These methods involve a more direct consideration of ways in which cycles in the graph can bound the faces of an embedding. Our second method involves creating an auxiliary graph, with vertices taken as particular cycles in the graph, and adjacency indicating when two such cycles cannot be taken simultaneously as faces of an embedding, and then using the independence number of the auxiliary graph to give an upper bound on the number of faces (and hence a lower bound on the minimum genus). According to Carsten Thomassen (in a private communication), this approach has not been taken before. Our third approach uses (mixed) integer linear programming to achieve the same thing when the auxiliary graph method is not helpful, and our fourth method uses integer linear programming directly for finding the faces of a minimum genus embedding of the graph.

All of these methods are quite general, in the sense that they do not expect the given graph to possess some non-trivial symmetry, even though we developed each of them to deal with graphs that do.

In particular, our new methods enabled us to prove the following:

(a) the minimum non-orientable genus of the Cartesian product graph $C_{3} \square C_{3} \square C_{3}$ is 13, answering a 1998 question by Brin and Squier [4],

(b) the minimum non-orientable genus of the Gray graph is 13 , complementing the determination in 2005 of its minimum orientable genus in [30],

(c) the minimum orientable genus of the Doyle-Holt graph is 5, answering a 2005 question by Marušič, Pisanski and Wilson [30],

(d) the minimum non-orientable genus of the Doyle-Holt graph is 8, complementing (c),

(e) the minimum orientable genus of the dual Menger graph of the Gray $\left(27_{3}\right)$ configuration is 6 , answering two more questions from [30], and its minimum non-orientable genus is 11 ,

(f) the minimum orientable genus of the second smallest semi-symmetric 3-valent graph (which has order 110) is 15 , answering the penultimate question in [30], and its minimum non-orientable genus is 28 , and

(g) the minimum orientable genus of the Ljubljana graph (which has order 112) is 13, answering the final question in [30], and its minimum non-orientable genus is 27 .

We also found the minimum orientable and non-orientable genera for several other interesting graphs, including the Folkman graph and Tutte's 8-cage.

Many of the discoveries mentioned above are described in this paper, in each case to illustrate the particular method(s) we used to make them. Before that, we give some further background in Section 2. Then we describe our 'subgroup orbit' method in Section 3, our 'independence number' approach in Section 4, and our integer linear programming approach in Section 5.

\section{Further background}

In this section we give further background on graph embeddings, known as maps, and we briefly describe their connection with geometric realisations of certain set systems, and also explain the use of voltage graphs to construct embeddings of particular kinds of graphs. 


\subsection{Graph embeddings}

By an embedding of a connected graph $X$ we mean a 2-cell embedding of $X$ on some closed surface $S$. In particular, such an embedding has the property that when the graph is removed from the surface $S$, it breaks up $S$ into simply-connected open regions (homeomorphic to open unit disks), called the faces of the embedding. (Note here that we do not require the closure of a face to be homeomorphic to a closed unit disk.) Such an embedding of a graph is also called a map, and then the graph $X$ is the 1-skeleton of the map $M$.

Next, if we denote the sets of vertices, edges and faces of the map $M$ by $V, E$ and $F$ respectively, then by the well known Euler-Poincaré formula we have

$$
|V|-|E|+|F|=\chi
$$

where $\chi$ is the Euler characteristic of the surface $S$. If $S$ is orientable, then $\chi=2-2 g$ where $g$ is the genus of $S$ (and of $M$ ), and in that case; furthermore, in the special case where $g=0$ (and $\chi=2$ ), the map $M$ is called planar or spherical, while if $g=1$ (and $\chi=0$ ) then $M$ is Euclidean or toroidal, and if $g>1$ (and $\chi<0$ ) then $M$ is hyperbolic. On the other hand, if $S$ is non-orientable, then $\chi=2-p$ where $p$ is the genus of $S$, with $p=1$ when $S$ is the projective plane, or $p=2$ when $S$ is the Klein bottle, and so on.

A given graph $X$ may have several different embeddings, and the Euler characteristic (and hence also the genus) of each one is determined by the number of resulting faces, since the numbers of vertices and edges are exactly the same as for the graph $X$. In the orientable case, the smallest and largest achievable values of the genus $g$ are called the minimum orientable genus and the maximum orientable genus of $X$, respectively. The minimum orientable genus is often called simply the genus of $X$, and denoted by $\gamma(X)$. Similarly, in the non-orientable case, the smallest and largest achievable values of $p$ are the minimum and maximum non-orientable genus of $X$, respectively. The former is sometimes also called the cross-cap number of $X$, and is denoted by $\bar{\gamma}(X)$. In both cases, the minimum genus occurs when the number of faces is maximised, or equivalently, when the average face-size is minimised.

As mentioned in the Introduction, every embedding of a connected graph $X$ on an orientable surface is uniquely determined by the cyclic orientation of the edges at each vertex, giving what is known as the 'rotation system' of the embedding. Equivalently, the embedding can be described by giving a set of closed walks (not necessarily simple cycles) bounding the faces, with consistent orientation and folding well around each vertex. For example, if the (anti-clockwise) rotations at the vertices 1 to 4 of $K_{4}$ are taken as those which induce the permutations $(2,3,4),(1,4,3),(1,2,4)$ and $(1,3,2)$ on their neighbours, respectively, and we trace faces anti-clockwise (by 'turning left' at each successive vertex, then the faces are bounded by the cycles $(1,2,3),(1,3,4),(1,4,2),(2,4,3)$, and this gives an orientable embedding of characteristic $\chi=4-6+4=2$ and minimum orientable genus 0 . If we then replace the rotation at vertex 4 by its inverse, then the faces are bounded by the cycle $(1,2,3)$ and the closed walk $(1,3,4,2,1,4,3,2,4)$, giving an orientable embedding with $\chi=4-6+2=0$ and maximum orientable genus 1 .

For non-orientable embeddings, the situation is a little more complicated. Any such embedding can also be described by cyclic orientation of the edges at each vertex, or by a set of closed walks bounding the faces, but without consistent orientation. For example, there exists a non-orientable embedding of $K_{5}$ with $\chi=5-10+6=1$ and minimum non-orientable genus 1 with faces bounded by the cycles $(1,3,5),(1,3,4),(2,4,3)$, 
$(1,5,2),(2,5,4),(1,4,5,3,2)$, and for this, the local orientations at vertices 1 to 5 are given up to reversal by the cyclic permutations $(2,5,3,4),(1,3,4,5),(1,4,2,5),(1,3,2,5)$ and $(1,2,4,3)$ of their neighbours, but there is no consistent way of orienting these cycles that gives an orientable embedding with the same face-bounding cycles. A connection between the two descriptions above can be made by 'twisting' some edges. Further details are explained in [19, 44] for example.

Finally, before continuing, we make two more points. One is that we may assume that the given connected graph $X$ has no vertices of valency 1 or 2 , as their presence does not affect the minimum (or maximum) genus of the graph: in any embedding, a leaf can be added to any vertex, and similarly, a new vertex of valency 2 can be inserted into any edge, without altering the genus. Another is that sometimes for ease of expression we will use $F_{k}$ to denote the number of faces of size/length $k$, and $F_{\ell}$ to denote the number of faces that are larger than some prescribed integer $k$.

\subsection{Connections with geometric realisations of block designs and configurations}

Closely related to the study of embeddings of graphs in surfaces is the study of geometric realisations of set systems, especially block designs and combinatorial configurations.

In 1897, Heffter observed that certain triangular embeddings of graphs in surfaces can be used to construct two-fold triple systems, with the role of the blocks being played by the faces of the map; see [23]. Subsequent work by others took this further, and showed a link between partially balanced incomplete block designs (PBIBDs) and triangular embeddings of strongly regular graphs, for example. Further details can be found in the surveys [16, 17].

A combinatorial configuration is a set system with intersection properties that mimic the properties of geometric configurations of points and lines, or occasionally configurations of other geometric objects such as circles, planes, and so on. Geometric realisations of configurations make up an important and classical area of geometry, described for example in books by Grünbaum [20], Hilbert and Cohn-Vossen [24] and Pisanski and Servatius [37]. Many authors consider embeddings of the Levi graph (incidence graph) of a configuration in a surface to be a geometric embedding of the configuration - see for example the work by Coxeter in [10]. Similarly, geometric realisations of neighbourhood geometries were considered by Van Maldeghem in [48].

On the other hand, any isometric embedding of a graph on a surface gives a geometric realisation of a point-circle configuration, by drawing a circle through the neighbourhood of each vertex of the graph. This was first observed by Gévay and Pisanski for the Euclidean plane [15], and later by Izquierdo and Stokes for other surfaces [46]. Note that this way of realising configurations geometrically is essentially different from the embeddings of block designs described above, because it is not the faces but rather the rotation systems of the embedded graph that constitute the blocks (or circles) of the geometric set system. In particular, isometric embeddings of Moore graphs induce geometric realisations of balanced pentagonal geometries, and this was the motivation for our initial work on embeddings of the Hoffman-Singleton graph, as explained in [46].

\subsection{Voltage graphs and covering graphs}

Voltage graphs provide a very good way to describe or construct covers of a given smaller graph (or multigraph), and can also be used to construct certain kinds of embeddings of such covering graphs. Here we give a brief summary of some key points about these things, 
and refer the reader to $[18,19]$ for further details.

Let $X$ be any finite graph whose automorphism group $A=\operatorname{Aut}(X)$ has a non-trivial subgroup $B$ that acts semi-regularly on $V(X)$ and $E(X)$, meaning that every non-trivial element of $B$ fixes no vertex or edge of $X$. In this case, all orbits of $B$ on $V(X)$ or $E(X)$ have the same length $n=|B|$. Then we may define a smaller graph $Y$ whose vertices are the orbits of $B$ on $V(X)$, and an edge joins two such vertices if and only if some edge of $X$ joins a pair of vertices in the corresponding orbits. In particular, $Y$ is a quotient of $X$, and $X$ is a regular cover of $Y$.

Now choose a set of representatives of the orbits of $B$ on $V(X)$, and let $\bar{v}$ be the representative of the $B$-orbit containing a vertex $v$. If $\{v, w\}$ is any edge of $X$, then so is $\left\{\bar{v}, \bar{w}^{\beta}\right\}$ for some $\beta \in B$, and hence so is $\left\{\bar{v}^{\alpha}, \bar{w}^{\beta \alpha}\right\}$ for all $\alpha \in B$. Accordingly, there is an arc from $\bar{u}^{B}$ to $\bar{v}^{B}$ in the quotient graph $Y$ that we can label with the element $\beta$ of $B$. (Also the reverse are could be labelled with $\beta^{-1}$, but that is not necessary.) After doing this for an edge from each orbit of $B$ on $E(X)$, we have a directed labelling of the edges of $Y$ that gives enough information to define the covering graph $X$ uniquely, with $B$ considered as a regular permutation group of degree $n=|B|$. When so labelled, the quotient graph $Y$ is called the voltage graph, and $B$ is called the voltage group, while $X$ is the derived graph, constructible from the graph $Y$ and the voltage assignments.

The vertex-set of the derived graph can be regarded as the Cartesian product $V(Y) \times B$, and its edges are of the form $\{(y, \alpha),(z, \beta \alpha)\}$ where $\alpha \in B$, and $(y, z)$ is an arc of $Y$ labelled with $\beta \in B$. To see the connection with constructing $Y$ from the derived graph $X$, note that $y$ and $z$ may be viewed as $\bar{v}$ and $\bar{w}$, and $(y, \alpha)$ as $v=\bar{v}^{\alpha}$, and $(z, \beta \alpha)$ as $\bar{w}^{\beta \alpha}$.

The voltage graphs described above are also called regular voltage graphs, and they correspond to regular coverings of graphs. Permutation voltage graphs were introduced by Gross and Tucker in [18], where they proved that it is enough to use permutations from a symmetric group as labels on the (possibly multiple) edges of a voltage graph, to represent an ordinary covering of a given graph. Any regular voltage graph can be expressed as a permutation voltage graph. More generally, a branched covering of a graph (which in the literature is also known as a wrapped quasi-covering of a graph (see [27, 36])) is a pair of graphs, similar to the pair consisting of a permutation voltage graph and its derived graph, except that branched (or wrapped) vertices are also allowed.

Next, embeddings of the voltage graph $Y$ can also be used to construct embeddings of the derived graph $X$. To do this, simply assign a cyclic rotation of the edges at each vertex of $Y$, and then use the voltage assignments to give the analogous rotations at the corresponding vertices of $X$.

One particularly good feature of this process is that it preserves much of the symmetry of the initial embedding - and indeed there are many cases where a highly symmetric or minimum genus embedding can be described in terms of a voltage graph (see [29]). Not all embeddings of the derived graph $X$ can be obtained in this way, however, as we will see with the Hoffman-Singleton graph. Given a nice embedding of a (branched) cover, it is not certain that the quotient of this embedding is an embedding which is easily recognisable as nice embedding for lifting. In other words, it is not usually clear in advance what kinds of embeddings of the voltage graph (or even what voltage groups and voltage assignments) will result in particularly nice embeddings of the derived graph.

In Section 3.4, we will compare one of our methods for finding graph embeddings with methods that use coverings and voltage graphs. 


\section{The subgroup orbit method}

Here we present the method that we used successfully to find minimum genus embeddings of many of the graphs mentioned in the Introduction. It works well for finding embeddings of a graph with certain degree of non-trivial symmetry. The method uses selected elements of the automorphism group of the graph to construct an embedding which will have an automorphism group featuring at least the selected automorphisms.

\subsection{Motivation}

This method was inspired by properties of regular maps.

A flag of a map $M$ is usually defined as an incident vertex-edge-face triple $(v, e, f)$ in $M$, but more technically it should be defined as follows, to avoid ambiguity in cases where an edge $e$ lies in just one face. Subdivide each face $f$ of length $k$ in $M$ into $2 k$ topological triangles, with the vertices of each triangle being the centre of the face $f$, a vertex $v$ of $M$ on the boundary of the face $f$, and the mid-point of an edge $e$ incident with both $v$ and $f$. We then call each such triangle a flag of $M$. In this way, every edge of $M$ lies in four flags (with two for each choice of the vertex $v$ ).

An automorphism of map $M$ is a bijection from $M$ to itself that preserves its vertexset, edge-set and face-set, and preserves incidence between these sets. By connectness, every automorphism of $M$ is uniquely determined by its effect on any flag, so the automorphism group of $M$ (denoted by $\operatorname{Aut}(M)$ ) acts semi-regularly on flags, and it follows that $|\operatorname{Aut}(M)|$ divides the number of flags, namely $4|E(M)|$.

A map $M$ is called regular if $\operatorname{Aut}(M)$ is transitive (and hence acts regularly) on the flags of $M$, or if $M$ is orientable and the group of all orientation-preserving automorphisms of $M$ acts regularly on the arcs of $M$; see [11] (or [9], for example). These two definitions are not equivalent (indeed the two cases are different, but not mutually exclusive). In both cases the automorphism group of $M$ has a single orbit on faces, and if the face-size is small enough then $M$ can be expected to be a minimum genus embedding of $X$. (For example, this always happens when all faces of $M$ are triangular.) There are also non-regular maps whose automorphism group has a small number of orbits on faces, and again if the faces are small, then these can give minimum genus embeddings of the underlying graph.

Our method finds minimum genus embeddings for which some non-trivial subgroup of the automorphism group of the graph induces a group of automorphisms of the map, usually with a small number of orbits on faces, when such a subgroup exists.

Before describing it, we repeat the observation that the smallest genus embeddings have the largest possible number of faces (in each of the orientable and non-orientable cases). Also we note the following.

Lemma 3.1. If $X$ is a connected finite graph of girth $g$, then in any embedding of $X$, every face has size at least $g$, and the number of faces is at most $2|E(X)| / g$.

Proof. The first conclusion is obvious, and the second follows by counting incident edgeface pairs, which shows that the sum of the sizes of all faces at most $2|E(X)|$.

The above observations show that it makes sense to consider cycles in the graph of relatively small length (either girth cycles, or 'almost' girth cycles) as possibilities for the closed walks bounding the faces of a small genus embedding. We also use subgroups of the automorphism group of the graph (of order dividing $4|E|$ ) to reduce the search space. 


\subsection{Description}

Our subgroup orbit method proceeds as follows, for the given connected graph $X$ :

Step 1. Find the set $\mathcal{C}$ of cycles of $X$ of small lengths of interest.

Step 2. Find the automorphism group of $X$ and its conjugacy classes of subgroups.

Step 3. For every representative subgroup $G$ of order dividing $4|E(X)|$ in $\operatorname{Aut}(X)$, taken in decreasing order of $G$,

(a) find the set $\mathcal{S}$ of orbits of $G$ on the cycles in $\mathcal{C}$,

(b) find subsets of $\mathcal{S}$ whose union forms the set of faces of an embedding of $X$,

(c) for each such subset, determine the orientability and genus of the resulting map.

Note that Step 3(b) requires checking that the union of the chosen subsets of $\mathcal{S}$ uses every edge exactly twice; in particular, the sum of the lengths of the cycles in the union must be $2|E(X)|$. Also, if some set $\mathcal{S}$ of orbits of $G$ on cycles produces an embedding of $X$, then $G$ will induce a subgroup of the automorphism group of the resulting map, so its order must divide $4|E(X)|$.

Step 3(b) also requires that the cycles incident with each vertex $v$ fold well around $v$, providing a cyclic permutation of the edges incident with $v$. Testing this can be achieved simply by constructing a 'local' graph, representing the vertex-figure on the neighbourhood $X(v)$ of $v$, with an edge between vertices $u$ and $w$ if and only if the union contains a cycle with edges $\{u, v\}$ and $\{v, w\}$, and then checking that this graph is a $k$-cycle, where $k=|X(v)|$ is the valency of $v$. The test for orientability in Step 3(c) then follows on easily from that. Also Step 3(b) can be sped up by use of a backtrack search, adding and removing $G$-orbits on cycles to and from a union of such orbits, with feasibility tests at each node of the search tree.

In practice, the length of time needed for Steps 1 and 2 is relatively small, while most of the time is required for Step 3. Also the time needed increases as the order of $G$ decreases, because the number of orbits of $G$ on $\mathcal{C}$ increases. But usually we do not conduct Step 3 for every class of subgroups. Indeed we stop the search if it finds an orientable embedding and/or non-orientable embedding of provably minimum genus, since there is then no need to proceed further, and in that case we have found such an embedding (or embeddings) with largest possible automorphism group. Also we can stop the search if it takes too long or requires too much memory, but in principle it can work even when the subgroup $G$ is trivial.

\subsection{Application to the Hoffman-Singleton graph}

The Hoffman-Singleton graph is the unique Moore graph of valency 7 and diameter 2, and hence has order $1+7+7 \cdot 6=50$ and girth 5 .

It has a very nice 'pentagons-and-pentagrams' construction (due to Robertson [41]), which may be described as follows: Take five pentagons $P_{1}, P_{2}, P_{3}, P_{4}, P_{5}$, with each $P_{i}$ having vertices $u_{i 1}, u_{i 2}, u_{i 3}, u_{i 4}$ and $u_{i 5}$ and edges $\left\{u_{i 1}, u_{i 2}\right\},\left\{u_{i 2}, u_{i 3}\right\},\left\{u_{i 3}, u_{i 4}\right\}$, $\left\{u_{i 4}, u_{i 5}\right\}$ and $\left\{u_{i 5}, u_{i 1}\right\}$, and five pentagrams (5-pointed stars) $Q_{1}, Q_{2}, Q_{3}, Q_{4}, Q_{5}$, with each $Q_{i}$ having vertices $v_{i 1}, v_{i 2}, v_{i 3}, v_{i 4}$ and $v_{i 5}$ and edges $\left\{v_{i 1}, v_{i 3}\right\},\left\{v_{i 3}, v_{i 5}\right\},\left\{v_{i 5}, v_{i 2}\right\}$, $\left\{v_{i 2}, v_{i 4}\right\}$ and $\left\{v_{i 4}, v_{i 1}\right\}$, and then add an edge from vertex $u_{i j}$ to vertex $v_{r s}$ whenever $s \equiv i r+j(\bmod 5)$. 
Equivalently, it may be constructed as the derived graph of a graph $T$ of order 10 whose vertices are $P_{1}, P_{2}, P_{3}, P_{4}, P_{5}, Q_{1}, Q_{2}, Q_{3}, Q_{4}$ and $Q_{5}$, with a loop at each vertex and an edge joining each of the 25 pairs of vertices $P_{i}$ and $Q_{j}$, and voltage group $\mathbb{Z}_{5}$ (under addition). In particular, this makes it a 5 -fold cover of $T$.

For ease of notation, we may re-label the vertices $u_{11}, u_{12}, u_{13}, u_{14}, u_{15}, u_{21}, u_{22}, \ldots$, $u_{55}$ as 1 to 25 , and the vertices $v_{11}, v_{12}, v_{13}, v_{14}, v_{15}, v_{21}, v_{22}, \ldots, v_{55}$ as 26 to 50 . Then for example, the neighbours of the vertex 1 are $2,5,27,33,39,45$ and 46 .

The Hoffman-Singleton graph is vertex-transitive. Indeed its automorphism group has order 252000 and is isomorphic to $\mathrm{P} \Sigma \mathrm{U}(3,5)$, which is a semi-direct product of the simple linear group PSU $(3,5)$ by a cyclic group of order 2 generated by the Frobenius automorphism of $\operatorname{GF}\left(5^{2}\right)$. The stabiliser of a given vertex $v$ is isomorphic to $S_{7}$, which acts faithfully on the neighbourhood of $v$. In particular, the graph is also arc-transitive, or symmetric.

An easy computation with the MAGMA system [2] shows that the automorphism group has 148 conjugacy classes of subgroups, of orders $1,2,3,4,5,6,7,8,9,10,12,14,16$, $18,20,21,24,25,32,36,40,42,48,50,60,72,80,96,100,120,125,144,168,200,240$, 250, 336, 360, 480, 500, 720, 1000, 1440, 2000, 2520, 5040, 126000 and 252000 (with many orders repeated). We can limit our attention to those of order dividing $4|E|=700$, that is, of order $1,2,4,5,7,10,14,20,25,50$ or 100 .

It is easy to check that there is no subgroup of order 50 that is complementary to the vertex-stabiliser, and hence the Hoffman-Singleton graph is not a Cayley graph. Moreover, it has no subgroup of order 175,350 or 700 , and hence has no subgroup that acts regularly on the edges or on the arcs of the graph, or on the flags of any embedding. In particular, the Hoffman-Singleton graph is not the underlying graph of a regular map, and this explains why we started thinking about different kinds of embeddings. We collect some of our findings in the following.

Proposition 3.2. The Hoffman-Singleton is not a Cayley graph, and is not the underlying graph of a regular map.

Next, by Lemma 3.1, an upper bound on the number of faces of any embedding is $350 / 5=70$, with the bound attained only when all faces are pentagonal.

We implemented our subgroup orbit method in MAGMA, and ran it on an Apple laptop. With $\mathcal{C}$ chosen as the set of all cycles of length 5 (of which there are 1260), it took only minutes to check and eliminate subgroups of order 20 or more, but the computation then slowed down considerably once it reached subgroups of order 10 . Because of this, we restricted the search to cyclic subgroups of prime order, and that led us to discover some minimum genus embeddings.

One of the first ones we found (taking only a few minutes in the restricted computation) uses ten orbits on $\mathcal{C}$ of a cyclic subgroup of order 7 in the automorphism group of the graph, generated by the automorphism $\alpha$ that induces the permutation

$$
\begin{aligned}
& (2,5,27,33,39,45,46)(3,26,10,12,37,7,49)(4,29,9,36,13,6,47) \\
& \quad(8,19,48,28,50,30,20)(11,40,38,14,35,17,43)(15,25,16,41,32,18,23) \\
& (21,34,44,22,31,24,42)
\end{aligned}
$$

on the re-labelled vertices. Note that this permutation does not act semi-regularly on the vertices, since it fixes the vertex 1 . The 70 faces of the embedding are bounded by the ten 
5-cycles
$(1,2,34,32,5)$,
$(2,3,29,26,28)$
$(2,3,35,17,47)$,
$(2,28,23,38,40)$,
$(2,34,13,12,47)$,
$(2,40,18,44,41)$,
$(3,35,32,8,48)$,
$(3,36,12,44,42)$,
$(4,30,16,34,31)$,
$(4,30,28,23,43)$,

and their images under non-trivial powers of the automorphism $\alpha$.

This embedding is non-orientable, since the Euler characteristic $\chi$ is $50-175+70=$ -55 , which is odd. In particular, it is a non-orientable embedding of minimum genus, and gives the cross-cap number of the graph as $2-\chi=57$. The embedding is illustrated in Figure 1, and also in [46].

At this point, we note that the resulting map admits an automorphism of order 7 (acting on the underlying graph in the same way as $\alpha$ above), and also that with the help of MAGMA it is not difficult to show that there are no other map automorphisms apart from powers of $\alpha$, and so the full automorphism group of this map has order 7 .

Another non-orientable embedding we found of the same genus uses 14 orbits of a cyclic subgroup of order 5 generated by the automorphism $\beta$ that induces the semi-regular permutation

$$
\begin{aligned}
& (1,6,12,19,22)(2,7,13,20,23)(3,8,14,16,24)(4,9,15,17,25) \\
& (5,10,11,18,21)(26,50,43,40,31)(27,46,44,36,32)(28,47,45,37,33) \\
& (29,48,41,38,34)(30,49,42,39,35) .
\end{aligned}
$$

The 70 faces of this embedding come from the orbits of the following 5-cycles:

$$
\begin{array}{lll}
(1,2,41,20,33), & (1,33,23,48,46), & (1,46,16,42,45), \\
(1,45,13,14,27), & (1,27,18,17,39), & (1,39,36,38,5), \\
(1,5,50,47,2), & (2,3,4,37,40), & (2,34,31,18,40), \\
(3,4,30,8,48), & (3,48,18,44,42), & (3,42,9,26,29), \\
(4,5,32,11,43), & (4,31,15,39,37) . &
\end{array}
$$

We later used linear programming (as we will describe in Section 5) to find a large number of non-orientable embeddings of minimum genus with trivial automorphism group, and some further computations using MAGMA showed that 7 is the largest order of the group of automorphisms of any such embedding.

We collect our findings in the following theorem.

Theorem 3.3. The minimum non-orientable genus of the Hoffman-Singleton graph is 57, and occurs for embeddings with 70 pentagonal faces. Moreover, the maximum order of a group of automorphisms of such an embedding of this graph is 7, and other possibilities for the order are 1 and 5 .

For orientable embeddings, an upper bound on the number of faces is 69 , potentially giving Euler characteristic $\chi=50-175+69=-56$ and genus 29. In theory, this could be achieved in a number of ways: ranging from 68 faces of length 5 and one of length 10 , to 64 faces of length 5 and five of length 6 . 


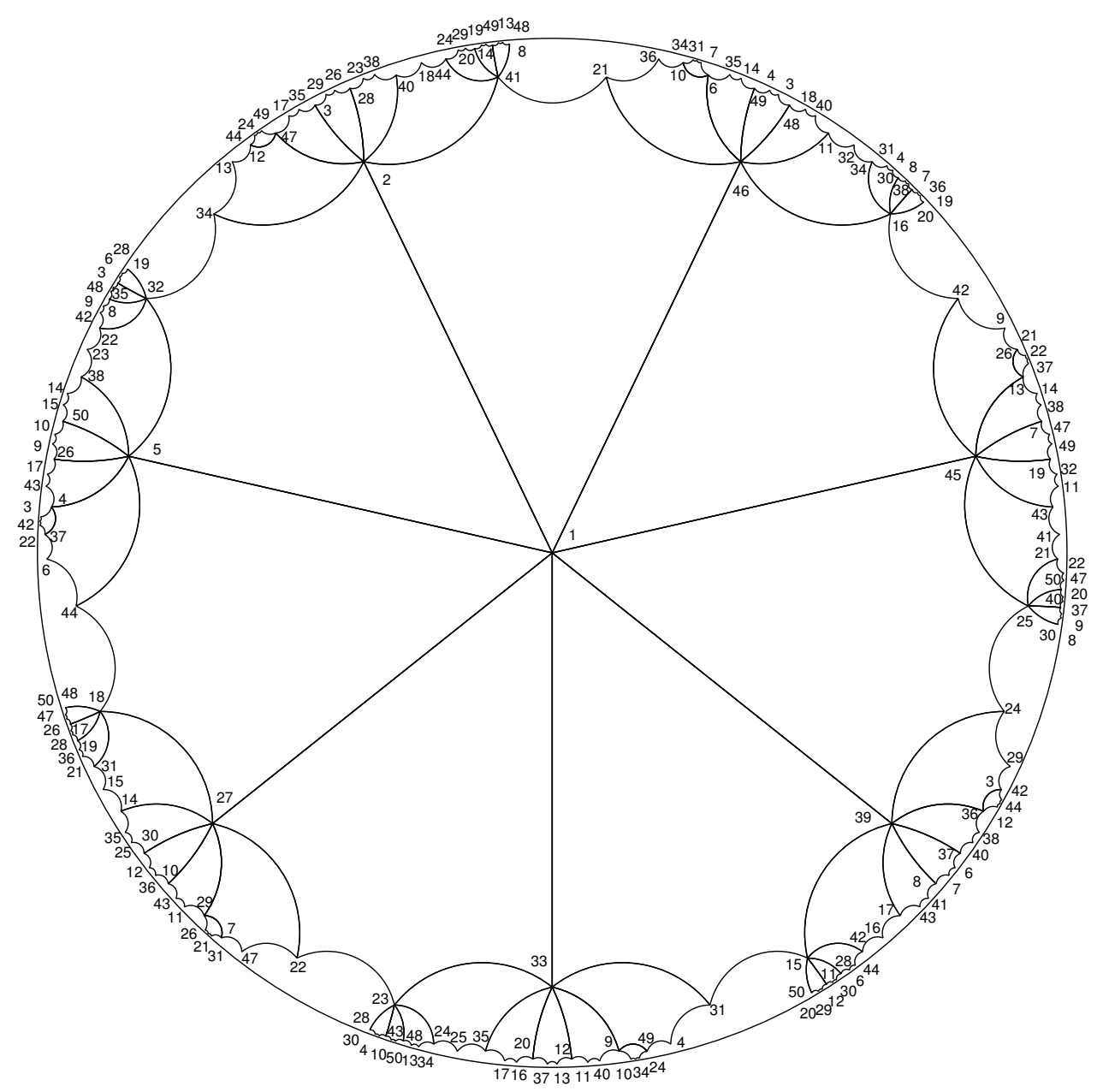

Figure 1: A minimum genus non-orientable embedding of the Hoffman-Singleton graph. 
We ran another version of our MAGMA procedure with $S$ chosen as a cyclic subgroup of order 5 in the automorphism group of the graph, and $\mathcal{C}$ chosen as the set of all cycles of length 5 and all the 6 -cycles in a single orbit of $S$, and found an orientable embedding of minimum genus 29, with 64 faces of length 5 and five of length 6 . This embedding came from the subgroup of order 5 generated by the obvious automorphism $\gamma$ of order 5 that induces the semi-regular permutation

$$
\begin{aligned}
& (1,2,3,4,5)(6,7,8,9,10)(11,12,13,14,15)(16,17,18,19,20) \\
& (21,22,23,24,25)(26,27,28,29,30)(31,32,33,34,35)(36,37,38,39,40) \\
& (41,42,43,44,45)(46,47,48,49,50) .
\end{aligned}
$$

This subgroup is not conjugate to the subgroup generated by the earlier automorphism $\beta$ mentioned above. The 69 faces of the embedding come from 11 orbits of $\langle\gamma\rangle$ of length 5 on 5-cycles, with representatives

$$
\begin{array}{lll}
(1,2,34,10,27), & (1,5,38,7,45), & (1,45,13,37,39), \\
(1,46,11,12,33), & (6,35,17,47,7), & (6,37,22,23,28), \\
(6,46,21,41,44), & (11,29,24,34,32), & (11,43,17,26,29), \\
(11,46,48,18,40), & (16,17,43,23,38), &
\end{array}
$$

plus another nine individual 5-cycles, which are all preserved by $\gamma$, namely

$$
\begin{array}{lll}
(6,7,8,9,10), & (11,15,14,13,12), & (16,20,19,18,17), \\
(21,25,24,23,22), & (26,28,30,27,29), & (31,33,35,32,34), \\
(36,39,37,40,38), & (41,43,45,42,44), & (46,49,47,50,48),
\end{array}
$$

and a single orbit of $\langle\gamma\rangle$ of length 5 on 6 -cycles, with representative

$$
(1,27,18,31,21,46) \text {. }
$$

These 69 cycles are consistent, in that they give the rotation system for an orientable embedding. For example, the seven of those 69 cycles that contain the vertex 1 are the six 5 -cycles

$$
\begin{array}{lll}
(1,2,34,10,27), & (5,1,33,9,26), & (1,5,38,7,45), \\
(2,1,39,8,41), & (1,45,13,37,39), & (1,46,11,12,33),
\end{array}
$$

plus the single 6 -cycle

$$
(1,27,18,31,21,46) \text {, }
$$

and these are consistent with $\rho=(2,39,45,5,33,46,27)$, which gives a rotation at vertex 1 . The rotations at other vertices can be found similarly.

The resulting orientable embedding admits $\gamma$ as a map automorphism of order 5 , and an easy MAGMA computation shows that there are no other automorphisms. In particular, the above embedding is chiral (irreflexible), meaning that it does not admit an orientationreversing automorphism. Also an extended MAGMA computation showed that 5 is the largest order of any group of automorphisms of an orientable embedding of minimum genus 29 .

We collect our findings in the following theorem. 
Theorem 3.4. The minimum orientable genus of the Hoffman-Singleton graph is 29, and this is attainable by a chiral embedding with 69 faces, of which 64 have length 5 and five have length 6, and with automorphism group of order 5 . Moreover, 5 is the maximum order of a group of automorphisms of any minimum genus orientable embedding of this graph.

\subsection{Comparison with the voltage graph method}

Here we make some observations that compare the voltage graph method (as described near the end of Subsection 2.3) with our new subgroup orbit method, in response to a suggestion by Tomaž Pisanski. Each of these two methods involves choice of an eventual group of automorphisms of an embedding (or just a suitable set of permutations), in order to reduce the size of the search space for nice embeddings, and this also makes it easy to describe each embedding found.

But there are many important respects in which they differ.

The voltage graph method involves guessing a way of regarding the given graph as a covering graph of a nice voltage graph (and then choosing suitable voltage assignments, and so on), while the subgroup orbit method does not do this, even though those things can sometimes be the outcome. In this sense, the subgroup orbit method is more systematic than the voltage graph method (even without putting any extra restrictions on the set $\mathcal{C}$ of cycles or the subgroup $G$, as we did when finding minimum genus orientable embeddings of the Hoffman-Singleton graph). Also the subgroup orbit method can find embeddings that are unlikely to be obtained by the voltage graph method. In particular, this may happen when vertices in some face are identified in the quotient embedding while others are not, but also in other cases where the quotient graph is not obvious or natural.

The minimum genus embeddings we found for the Hoffman-Singleton graph make a good illustration of these arguments. Our orientable embedding with 69 faces can be constructed from an embedding of the quotient graph via the automorphism $\gamma$ of order 5 , and this graph happens to be the very nice voltage graph $T$ from which the Hoffman-Singleton graph is often constructed (as described at the beginning of Section 3.3). On the other hand, the minimum genus non-orientable embeddings that we found have automorphisms that define quite different voltage graphs: the automorphism $\beta$ of order 5 gives a quotient graph on 10 vertices with multiple edges but no loops, while the automorphism $\alpha$ of order 7 defines a quotient graph on 8 vertices (with one being a branched vertex). Every embedding of one of these quotient graphs will give an embedding of the Hoffman-Singleton graph in some surface. In the third case (using $\alpha$ ), however, the quotient graph is not the most obvious one to choose. Also the third case also shows that no particular difficulties need arise from using an automorphism that is not semi-regular.

The next point we make is that it can be difficult to choose the quotient graph and voltage assignments when we want control over the size of the faces and/or the number of faces in the derived embedding, which of course is what we need to do when searching for minimum genus embeddings. Indeed it can be difficult even to guess what lengths the faces should have in the quotient embedding in order to get faces of the desired lengths in the covering graph, without considering also the values of the voltages on the edges, and how they compose. For example, some of the pentagonal faces of the non-orientable embedding obtained from the automorphism $\beta$ are lifted from closed walks of length 5 in the quotient embedding, consisting of a triangular face together with a closed walk of length 2 , but in the voltage graph construction it would not be immediately clear if such a walk would lift to a pentagonal face, or to something larger. In other examples, it may be easy to see how 
short closed walks will lift in the derived graph, and often they unwind simply as desired (almost by pure luck), but in many cases the situation can be rather complicated, especially when a face is created from a union of smaller closed walks.

Here we feel it is interesting to note that voltage graph methods cannot be used to construct a minimum non-orientable genus embedding of the Hoffman-Singleton graph from the natural 10-vertex voltage graph $T$ (mentioned earlier). Such an embedding must have 70 pentagonal faces, lifted from 14 closed walks of length 5 in $T$ that use each arc exactly once. According to [21], there are four types of cycles of length 5 in the HoffmanSingleton graph. Cycles of type I are lifted loops, cycles of type II and III are lifts of closed walks of type $(v, v, v, u, u)$, and cycles of type IV are lifts of a cycle of length 4 with an attached loop. Any walk that lifts to a cycle may use each arc no more than once, and it follows that only cycles of types I and IV can be used in a lifted embedding. (Any closed walk of type $(v, v, v, u, u)$ in $T$ could not unwind to a simple cycle of length 5 in the derived graph if the loop at $v$ was taken in both possible directions, and so would have to traverse the loop at $v$ twice in the same direction.) On the other hand, a counting argument shows that we cannot cover each arc in $T$ exactly once using quotient walks of cycles of type I and IV, and so this voltage graph $T$ cannot be used to construct an embedding of the Hoffman-Singleton graph with only pentagonal faces.

The above example shows that the knowledge of a 'special' voltage graph does not necessary help when looking for a minimum genus embedding. More generally, if a voltage graph has a large number of vertices or edges, then it can be quite a challenge to find nice embeddings of it, let alone nice embeddings of the derived graph, while the subgroup orbit method is quite capable of easily finding nice embeddings also in those cases. In summary, the subgroup orbit method can produce a greater range of embeddings than the voltage graph method.

On the other hand, the subgroup orbit method works best when the graph has nice embeddings with non-trivial symmetry, while the voltage graph method can be made to work well also in cases where that does not happen (using permutation voltage graphs).

\subsection{Some other examples}

Example 3.5. The Cartesian product $C_{3} \square C_{3} \square C_{3}$.

This is an arc-transitive graph of order 27 , valency 6 , girth 3 and diameter 3 (and is a Cayley graph for the abelian group $\mathbb{Z}_{3} \oplus \mathbb{Z}_{3} \oplus \mathbb{Z}_{3}$ ). By Lemma 3.1, any embedding of this graph has at most $162 / 3=54$ faces. In 1985 it was shown to have a genus 7 orientable embedding with 42 faces, by Mohar, Pisanski, Škoviera and White [32], and three years later Brin and Squier proved in [4] that any embedding has as most 43 faces, and thereby showed that the minimum orientable genus of $C_{3} \square C_{3} \square C_{3}$ is 7 , but they left open the question of the minimum non-orientable genus.

With a natural vertex-labelling, our subgroup orbit method implemented in MAGMA takes only a couple of minutes to produce a different and more symmetric orientable embedding of minimum genus than the one found in [32]. This new embedding has automorphism group $S$ of order 36 , generated by elements that induce the permutations

$(2,7)(3,4)(5,9)(10,19)(11,25)(12,22)(13,21)$

$(14,27)(15,24)(16,20)(17,26)(18,23)$ 
and

$$
\begin{aligned}
& (1,14,27)(2,15,25)(3,13,26)(4,23,21,10,17,9) \\
& (5,24,19,11,18,7)(6,22,20,12,16,8) .
\end{aligned}
$$

The resulting map has 18 triangular faces, 18 quadrangular faces and 6 hexagonal faces, coming from the orbits under $S$ of the 3 -cycles $(4,6,5)$, the 4 -cycle $(1,2,8,7)$ and the 6 -cycle $(2,3,21,24,23,5)$. In particular, the first of the two generators for $S$ given above reverses the 4 -cycle $(1,2,8,7)$, and it follows that this embedding is reflexible.

Our subgroup orbit method also quickly finds a non-orientable embedding of minimum genus, with 43 faces, answering the question left open in 1988 by Brin and Squier [4]. This embedding has 24 triangular faces, 12 quadrangular faces, and 7 hexagonal faces, and its automorphism group is a dihedral group of order 12 , generated by two elements that induce the permutations

$$
\begin{aligned}
& (2,3)(4,7)(5,9)(6,8)(10,19)(11,21)(12,20)(13,25) \\
& (14,27)(15,26)(16,22)(17,24)(18,23)
\end{aligned}
$$

and

$$
\begin{aligned}
& (1,5,9)(2,8,7,4,6,3)(10,14,18)(11,17,16,13,15,12) \\
& (19,23,27)(20,26,25,22,24,21) .
\end{aligned}
$$

The 43 faces come from the orbits of the cycles $(1,2,3),(2,11,20),(10,11,12)$, $(1,2,11,10),(10,12,15,24,22,19)$ and $(2,3,6,4,7,8)$.

Thus we have proved the following improvement of what was achieved in [32] and [4].

Theorem 3.6. The minimum orientable genus of the Cartesian product $C_{3} \square C_{3} \square C_{3}$ is 7, and this is attainable by a reflexible embedding with 42 faces, in which there are 18 faces of length 3, plus 18 of length 4, and 6 of length 6, and with automorphism group of order 36 . The minimum non-orientable genus of the Cartesian product $C_{3} \square C_{3} \square C_{3}$ is 13, and this is attainable by an embedding with 43 faces, in which there are 24 faces of length 3, plus 12 of length 4, and 7 of length 6, and with automorphism group of order 12.

Example 3.7. Tutte's 8-cage.

This is the smallest 5-arc-transitive 3 -valent graph. It is bipartite of order 30, with girth 8 ; indeed it is also the smallest 3 -valent graph of girth 8 . Its automorphism group is isomorphic to $\operatorname{Aut}\left(S_{6}\right)$, of order 1440 .

The number of faces of any embedding is bounded above by $\lfloor 2|E| / 8\rfloor=\lfloor 90 / 8\rfloor=11$. Moreover, if there are exactly 11 faces, and $F_{8}$ and $F_{\ell}$ are the numbers of faces of length 8 and greater than 8 , then $88+2 F_{\ell}=8\left(F_{8}+F_{\ell}\right)+2 F_{\ell}=8 F_{8}+10 F_{\ell} \leq 2|E|=90$ and so $F_{\ell} \leq 1$, which implies that there are ten faces of length 8 and one of length 10 .

Our subgroup orbit method quickly gives a minimum genus non-orientable embedding with 11 faces, and cyclic automorphism group of order 10. With a suitable labelling of vertices, the automorphism group is generated by an element inducing the permutation

$$
\begin{aligned}
& (1,11,25,20,26,3,23,22,28,14)(2,5,13,29,19,7,15,24,12,6) \\
& \quad(4,27,17,8,18,9,16,10,21,30)
\end{aligned}
$$


and the ten faces of length 8 come from the orbit of $(1,2,5,11,23,16,8,4)$, while the single face of length 10 is bounded by the cycle $(2,6,12,24,15,7,19,29,13,5)$.

Also our subgroup orbit method gives an orientable embedding with 9 faces, and automorphism group of order 3. The automorphism group $S$ is generated by an element inducing the permutation

$$
\begin{array}{r}
(2,3,4)(5,9,10)(6,7,8)(11,21,18)(12,19,16) \\
(13,17,22)(14,15,20)(23,28,26)(24,29,30),
\end{array}
$$

and the embedding has three faces of length 8 , three of length 10 and three of length 12 , which come from the orbits under $S$ of the cycles

$$
\begin{aligned}
& (1,2,5,11,23,16,8,4), \quad(5,13,25,17,30,14,26,18,27,11) \quad \text { and } \\
& (2,6,14,30,16,23,15,7,19,29,13,5) .
\end{aligned}
$$

Our method found no orientable embedding with 11 faces, for a good reason. If there existed one, then there would be ten faces of length 8 and a single face of length 10 (as shown above). By transitivity of the automorphism group of Tutte's 8-cage on 10-cycles, we may choose any 10-cycle $C$ to bound the single face of length 10 , and then consider the way the other ten faces wrap around it. By inspection of the edge-set of the graph, it is easy to see that there are exactly four possibilities for a cycle of length 8 containing any edge, and it follows that there are $4^{10}$ possibilities for how to arrange potential faces of this length around the given 10-cycle $C$. But then an easy MAGMA computation shows that in all $4^{10}$ cases, some arc is repeated in two different faces, so this is impossible. (In fact there are only two embeddings that can be found in this way, and both are non-orientable.)

Thus we have the following:

Theorem 3.8. The minimum orientable genus of Tutte's 8-cage is 4, attainable by a chiral embedding with 9 faces, in which there are three faces of length 8 , three of length 10 , and three of length 12, and with automorphism group of order 3 . The minimum non-orientable genus of Tutte's 8-cage is 6, attainable by an embedding with 11 faces, in which there are ten faces of length 8 and one of length 10, and with cyclic automorphism group of order 10.

Further examples will be met in the next two sections.

\section{The independence number approach}

\subsection{Motivation and description}

Lemma 3.1 gives a theoretical upper bound on the number of faces of an embedding, and hence a lower bound on the minimum genus. If an embedding attains that bound, then it will automatically have minimum genus (whether orientable or not). Also if an orientable embedding falls short by just one face, then it will have minimum orientable genus, since in that case the Euler characteristic has to be even.

The two examples considered in Subsection 3.5 (and many other graphs besides those) show that these theoretical upper bounds on the number of faces of an embedding are not always attainable, and in such cases, some other information is required to help decide whether a given embedding has minimum genus. This was already done for $C_{3} \square C_{3} \square C_{3}$ (in Example 3.5) by Brin and Squier [4], using knowledge of the structure of the graph to reduce the bound from 54 to 43 faces, and similarly, in Example 3.7 we used some 
particular properties of Tutte's 8-cage to decrease the bound from 11 to 9 in the orientable case. These kinds of approach, however, are not likely to work well in general, so some other approaches are needed.

The main idea of our new approach is that we analyse an appropriate set $\mathcal{C}$ of cycles of the graph that are candidates for the faces, with the aim of finding an upper bound on the number of members of $\mathcal{C}$ that can be combined together to form the faces of an embedding or partial embedding. For example, the set $\mathcal{C}$ could be the set of all girth cycles, or all cycles of length close to the girth.

We then define an auxiliary graph $X_{\mathcal{C}}$ with $\mathcal{C}$ as its vertex-set, and with two cycles in $\mathcal{C}$ joined by an edge if and only they cannot occur together in the same embedding.

There are several ways of telling that two cycles cannot occur in the same embedding. Here we use the fact that the local arrangement of neighbours of a vertex requires that any given 2-path lies in at most one face (under the assumption that no vertex of $X$ has valency 2 ), and accordingly, we define an edge between two members of $\mathcal{C}$ if and only if they have a 2-path in common.

Next, we compute the independence number of the auxiliary graph $X_{\mathcal{C}}$. This is the maximum number of pairwise non-adjacent vertices of $X_{\mathcal{C}}$, and can be found (for example) in MAGMA using the MaximumIndependent Set command. The resulting number gives an upper bound on the number of cycles from $\mathcal{C}$ that can bound faces of an embedding, and hence can be used to find a lower bound on the average face size, and thereby obtain an improved upper bound on the total number of faces.

The method can be summarised as follows:

Step 1. Choose an appropriate set $\mathcal{C}$ of cycles of interest in the given graph $X$.

Step 2. Define the auxiliary graph $X_{\mathcal{C}}$ on the vertex-set $\mathcal{C}$, with two elements of $\mathcal{C}$ joined by an edge if and only if they cannot occur together in the same embedding.

Step 3. Find the independence number of the auxiliary graph $X_{\mathcal{C}}$, which gives an upper bound on the number of the cycles of $\mathcal{C}$ that can occur as faces of any embedding.

This approach works for both orientable and non-orientable embeddings alike, but can be further improved for orientable embeddings by taking $\mathcal{C}$ as a suitable set of oriented cycles, and by joining two elements of $\mathcal{C}$ by an edge when they have either an $\operatorname{arc}$ (ordered edge) or an underlying 2-path (or both) in common.

Also at Step 3 in both cases, the MaximumIndependent Set command can produce an independent set of maximum size, in case that is helpful.

As the examples below will show, this approach can lead to significant reduction in the upper bound on the number of faces, and then help with determining the minimum genus.

\subsection{Some applications}

\section{Example 4.1. The Gray graph.}

This is the smallest cubic (3-valent) graph that is semi-symmetric, which means regular and edge-transitive but not vertex-transitive; see [8]. It is bipartite with order 54, diameter 6 and girth 8 , and has automorphism group of order 1296. An upper bound on the number of faces of any embedding is $\lfloor 162 / 8\rfloor=20$, but this is not sharp. The minimum orientable genus of the Gray graph was found in 2005 by Marušič, Pisanski and Wilson [30] to be 7 , via an embedding with only 15 faces, obtained from the embedding of $C_{3} \square C_{3} \square C_{3}$ on a surface of genus 7 given in [32]. 
Our new approach also gives both this and the minimum non-orientable genus quite easily. First, the Gray graph has 81 cycles of length 8 , but none of length 10 . With $\mathcal{C}$ taken as the set of all 8-cycles, our independence number approach gives $F_{8} \leq 9$, and then because there are no 10 -cycles, all other faces have length at least 12 , and so we find that $|F| \leq 9+(2|E|-72) / 12=9+90 / 12$, which gives $|F| \leq 16$. Hence every non-orientable embedding of the Gray graph has at most 16 faces, while every orientable embedding has at most 15 .

Furthermore, our subgroup orbit method easily finds one of each kind of embedding from a dihedral subgroup $S$ of order 6 in the automorphism group of the graph: an orientable embedding with $F_{8}=F_{12}=6$ and $F_{14}=3$, and a non-orientable embedding with $F_{8}=9, F_{12}=4$ and $F_{14}=3$.

With a suitable labelling of the vertices, the dihedral subgroup $S$ can be generated by the two elements of orders 2 and 3 inducing the permutations

$$
\begin{aligned}
& (1,2)(3,15)(5,14)(6,8)(7,9)(11,13)(12,17)(16,18)(19,21)(20,27) \\
& (23,24)(25,26)(29,31)(30,32)(34,35)(36,37)(38,40)(39,47)(42,43) \\
& (44,45)(46,48)(49,53)(50,52)(51,54)
\end{aligned}
$$

and

$$
\begin{aligned}
& (1,2,4)(3,9,11)(5,6,17)(7,15,13)(8,14,12)(10,27,20)(16,26,23) \\
& (18,24,25)(19,21,22)(29,32,34)(30,31,35)(33,47,39)(36,45,42) \\
& (37,43,44)(38,40,41)(46,52,54)(48,51,50),
\end{aligned}
$$

and then the faces of the orientable embedding come from the orbits of $S$ containing the 8-cycle

$$
(3,29,8,42,23,46,10,33)
$$

the 12-cycles

$$
\begin{aligned}
& (1,29,3,36,14,41,5,37,15,31,2,28), \\
& (1,30,5,41,22,51,27,52,21,40,8,29),
\end{aligned}
$$

and the 14-cycle

$$
(3,33,15,37,18,53,25,51,22,54,26,49,16,36),
$$

while those of the non-orientable embedding come from the orbits of $S$ containing the 8-cycles

$$
(1,28,2,31,15,33,3,29), \quad(3,33,10,46,23,49,16,36),
$$

the 12-cycles

$$
\begin{aligned}
& (5,37,18,50,19,38,12,45,26,54,22,41), \\
& (10,48,21,52,27,51,22,54,20,50,19,46),
\end{aligned}
$$

and the 14-cycle

$$
(1,29,8,42,11,34,12,38,6,31,15,37,5,30) \text {. }
$$


The above orientable embedding is reflexible, since the 12-cycle

$$
(1,29,3,36,14,41,5,37,15,31,2,28)
$$

is inverted by conjugation by the first generator of $S$.

Thus we have the following improvement of what was found in [30].

Theorem 4.2. The minimum orientable genus of the Gray graph is 7, attainable by a reflexible embedding with 15 faces, of which six have length 8 , six have length 12 , and three have length 14, and with dihedral automorphism group of order 6 . The minimum non-orientable genus of the Gray graph is 13, attainable by an embedding with 16 faces, in which nine have length 8 , four have length 12 , and three have length 14, and with the same automorphism group of order 6 as in the orientable case above.

Example 4.3. The Ljubljana graph.

This is the third smallest semi-symmetric cubic graph. It is believed to have been first found by R. M. Foster in the 1970s, and first mentioned in [3]. It was later rediscovered in [5], as well as in the computations that produced the list of all small semi-symmetric 3 -valent graphs published in [8]. It is bipartite with order 112, diameter 8 and girth 10, and has soluble automorphism group of order 168. Other properties of this graph are described in [7].

The upper bound on the number of faces of any embedding given by Lemma 3.1 is $\lfloor 336 / 10\rfloor=33$, but we can reduce this to 32 using our independence number approach.

If we take $\mathcal{C}$ as the set of all unoriented 10-cycles in the graph (of which there are 168), then the auxiliary graph $X_{\mathcal{C}}$ has independence number 24 , and so $F_{10} \leq 24$. Next, since the graph is bipartite, every other face has length 12 or more, and so counting incident edgeface pairs gives $336=2|E| \geq 10 F_{10}+12\left(|F|-F_{10}\right)=12|F|-2 F_{10} \geq 12|F|-48$, and it follows that $|F| \leq(336+48) / 12=384 / 12=32$. Also if there are exactly 32 faces, with 24 of length 10 , then the inequality becomes an equality, and then the other eight faces must all have length 12 .

Our subgroup orbit method provides an orientable embedding with exactly 32 faces, and automorphism group of order 24, isomorphic to $A_{4} \times C_{2}$. In particular, this embedding has minimum orientable genus.

With a suitable labelling of the vertices, the automorphism group $S$ can be generated by the elements of orders 2 and 3 inducing the permutations

$$
\begin{aligned}
& (1,39)(2,56)(3,52)(4,45)(5,42)(6,34)(7,15)(8,48)(9,51)(10,30)(11,14) \\
& (12,46)(13,33)(16,43)(17,47)(18,54)(19,31)(20,24)(21,44)(22,41) \\
& (23,29)(25,50)(26,28)(27,40)(32,49)(35,38)(36,55)(37,53)(57,108) \\
& (58,83)(59,89)(60,110)(61,112)(62,104)(63,100)(64,106)(65,75)(66,71) \\
& (67,78)(68,86)(69,94)(70,95)(72,87)(73,93)(74,99)(76,88)(77,111) \\
& (79,82)(80,102)(81,109)(84,98)(85,101)(90,105)(91,107)(92,96)(97,103)
\end{aligned}
$$


and

$$
\begin{aligned}
& (1,50,36)(2,26,13)(3,18,17)(4,38,37)(5,21,41)(6,45,46)(7,19,49) \\
& (8,10,27)(9,35,31)(11,51,12)(14,32,20)(15,53,23)(16,39,55) \\
& (22,30,56)(24,34,29)(28,54,44)(33,40,52)(42,47,48)(57,103,81) \\
& (58,85,90)(59,93,80)(60,75,88)(61,74,64)(62,91,68)(63,82,77) \\
& (65,107,92)(66,96,95)(67,108,102)(70,100,110)(71,86,111)(72,78,98) \\
& (73,97,101)(76,79,104)(83,109,84)(87,89,105)(94,99,112),
\end{aligned}
$$

and then the 32 faces of the orientable embedding come from the orbits of $S$ containing the 10-cycle

$$
(1,57,2,61,18,91,35,79,11,59)
$$

and the 12-cycle

$$
(2,57,4,63,10,78,34,95,41,80,12,60) .
$$

Also the first of the two generators above reverses orientation, so this embedding is reflexible.

Next, by applying a 'twist' to a single edge that is common to the boundary of two distinct faces, we can merge those two faces into one, and thereby obtain a non-orientable embedding with 31 faces (with $F_{10}=22, F_{12}=8$ and $F_{20}=1$, or $F_{10}=23, F_{12}=7$ and $F_{22}=1$, or $F_{10}=24, F_{12}=6$ and $F_{24}=1$ ). In all cases, the embedding has trivial automorphism group, or in other words, is asymmetric.

It turns out that all of the latter embeddings have minimum non-orientable genus, because there is just one embedding with 32 faces, namely the orientable one described above. To see this, we can use our independence number approach a slightly different way.

First, an easy MAGMA computation shows that the set $\mathcal{C}$ of all 168 cycles of length 10 in the Ljubljana graph forms a single orbit under the action of its automorphism group. Now take any one of these 10-cycles as a representative of $\mathcal{C}$, say $C$, and let $\mathcal{I}$ be the set of all independent 24-sets in the auxiliary graph $X_{\mathcal{C}}$ that contain $C$.

Next, partition the remaining 167 cycles from $\mathcal{C}$ into three subsets: forgettable 10 cycles, which lie in no 24 -set in $\mathcal{I}$, standard 10 -cycles, which lie in exactly one set in $\mathcal{I}$, and special 10 -cycles, which lie in more than one set in $\mathcal{I}$. An easy computation shows that there are 82 forgettable 10 -cycles, plus 63 standard 10 -cycles, and just $167-(82+63)=22$ special 10-cycles. The forgettable 10-cycles can be ignored, as they cannot bound any face in a 32-face embedding. and so we need only deal with the standard and special 10-cycles.

We do this by considering the ways in which a 2 -subset of $\mathcal{C}$ can be extended to an independent 24 -set in the auxiliary graph $X_{\mathcal{C}}$. Note that every independent 24 -set in $\mathcal{I}$ must contain a standard 10 -cycle $D$, since there are only 22 special 10 -cycles. Furthermore, there is just one set 24 -set in $\mathcal{I}$ containing a given standard 10 -cycle $D$, and hence just one independent 24 -set containing $\{C, D\}$. It follows that we can find all members of $\mathcal{I}$ by letting $D$ run through the set of 63 standard 10-cycles, and for each one, determining the largest independent subset of the induced subgraph of $X_{\mathcal{C}}$ on the set of 10-cycles in $\mathcal{C}$ that are independent of $C$ and $D$. When this subset has size 22 , its union with $\{C, D\}$ is a member of $\mathcal{I}$, and conversely, every member of $\mathcal{I}$ can be found in this way.

In fact, by a MAGMA computation we find that the set $\mathcal{I}$ has only five members, with each standard 10-cycle lying on just one of them, as follows:

- one containing 3 standard 10-cycles and 20 special 10-cycles, 
- one containing 11 standard 10-cycles and 12 special 10-cycles,

- one containing 12 standard 10-cycles and 11 special 10-cycles,

- one containing 18 standard 10-cycles and 5 special 10-cycles, and

- one containing 19 standard 10-cycles and 4 special 10-cycles.

A further MAGMA computation shows that the first one gives rise to our known orientable embedding of the Ljubljana graph (with 32 faces), while the other four are mutually equivalent under the action of the full automorphism group of the graph, and give rise to embeddings with fewer than 31 faces. Further details are available if necessary from the first author on request.

In particular, there is just one embedding of the Ljubljana graph with 32 faces, namely the orientable embedding we described earlier, and therefore every non-orientable embedding has at most 31 faces, and $|F|=31$ gives the minimum non-orientable genus.

Hence we have answered the final question from [30], by proving the following.

Theorem 4.4. The minimum orientable genus of the Ljubljana graph is 13, attainable by a reflexible embedding with 32 faces, of which 24 have length 10 and eight have length 12 , and with automorphism group of order 24 isomorphic to $A_{4} \times C_{2}$. The minimum nonorientable genus of the Ljubljana graph is 27 , attainable by an embedding with 31 faces, and trivial automorphism group.

\section{Use of integer linear programming}

\subsection{Background and description}

Our independence number approach can be regarded as a constraint satisfaction problem on a subset of the cycles of the graph, in the sense that it finds the maximum number of cycles from the given set $\mathcal{C}$ that can be considered as bounding cycles for the faces of some embedding. This approach can also be modelled as an integer linear programming (ILP) problem, by using variables $x_{C}$ for cycles $C \in \mathcal{C}$, with $x_{C}=1$ if $C$ is included as a bounding cycle, or 0 if not, and then maximising $\sum_{C \in \mathcal{C}} x_{C}$ subject to appropriate constraints.

This ILP variant is related to the successful use of the Kramer-Mesner method in the search for block designs, as shown in [28] for example. Incidentally, ILP has been used also to find planar embeddings of graphs [33], and drawings with minimum crossing number in the plane [6]. Also at about the same time as we were using ILP for graph embeddings and beginning to write up this work, another method using ILP was developed by Beyer, Chimani, Hedtke and Kotrbčík [1], but the latter method differs from our one, and we consider our method (and its variants) to be simpler.

In fact we developed four different ILP methods. The first two are particularly easy to describe, and are used to provide lower bounds on the minimum genus of a graph. The other two are modifications of the first two, and are used to construct actual embeddings of a graph in a surface.

In all of them, we will assume that the given connected graph $X$ has no vertices of degree 1 or 2 , and that $X$ is is bridgeless (or in other words, 2-edge-connected), so that in any embedding of $X$, every edge lies in two different faces. Also we use the term 2-arc for 
an ordered triple $(u, v, w)$ of vertices such that $u$ and $w$ are neighbours of $v$ in $X$, and the term 2-path for the same triple when the order of $u$ and $w$ is unimportant.

We now describe our first ILP method, for producing helpful information about the faces of embeddings of a given graph $X$, whether orientable or not. For this, we let $\mathcal{C}_{e}$ be the set of all cycles in the set $\mathcal{C}$ containing a given edge $e$, and $\mathcal{C}_{p}$ be the set of all cycles in $\mathcal{C}$ containing a given 2 -arc $p=(u, v, w)$ or its reverse $(w, v, u)$.

Step 1. Choose a suitable set $\mathcal{C}$ of unoriented cycles of interest in the given graph $X$, and define a variable $x_{C}$ for each cycle $C \in \mathcal{C}$, with $x_{C}$ to take the value 1 if $C$ is included as a bounding cycle of some face of the embedding, or 0 if not.

Step 2. Define the objective function as an linear combination of the variables $x_{C}$ with appropriate integer coefficients.

Step 3. Set up the constraints as follows:

- $x_{C} \in\{0,1\}$ for all $C \in \mathcal{C}$,

- $\sum_{C \in \mathcal{C}_{e}} x_{C} \leq 2$ for every edge $e$ of $X$, and

- $\sum_{C \in \mathcal{C}_{p}} x_{C} \leq 1$ for every 2-path $p$ in $X$.

Step 4. Find the maximum value of the objective function subject to the given constraints.

This gives an upper bound on the number of faces that can be bounded by the cycles in $\mathcal{C}$ in any embedding. For example, if the objective function is the sum $\sum_{C \in \mathcal{C}} x_{C}$ then the algorithm will search for the maximum number of cycles in the graph satisfying the constraints. Since the faces of any embedding must satisfy these constraints, this gives a simple computational method for bounding the number of faces in an embedding from above. Note that the objective function does not need to be the simple sum $\sum_{C \in \mathcal{C}} x_{C}$; indeed in the first example below, we take it as a non-trivial weighted combination of the variables $x_{C}$ for the cycles of interest in $\mathcal{C}$. Also the bound obtained might be less than the number of faces in a minimum genus embedding, for example when we are interested in what is possible for a partial embedding.

Our second ILP method is a modification of the first one, for orientable embeddings only, and the same comments as above apply to it. Steps 1,2 and 4 are the same, except that $\mathcal{C}$ is taken as a set of oriented cycles of interest, and Step 3 is similar to the one above, but we let $\mathcal{C}_{a}$ be the set of all oriented cycles in the set $\mathcal{C}$ containing a given $\operatorname{arc} a=(v, w)$, and then set up the constraints as

- $x_{C} \in\{0,1\}$ for all $C \in \mathcal{C}$,

- $\sum_{C \in \mathcal{C}_{a}} x_{C} \leq 1$ for every arc $a$ in $X$, and

- $\sum_{C \in \mathcal{C}_{p}} x_{C} \leq 1$ for every 2-path $p$ in $X$. 
Note that these two methods are designed to help provide good upper bounds on the number of faces of an embedding, but are not designed to actually find a minimum genus embedding. Some times they do find one, but as with the independence number approach, it can happen that an optimum solution to the ILP problem does not produce even a partial embedding, because the constraints are necessary but not sufficient. Nevertheless the methods can be very helpful, as the examples in the next subsection will show.

Our other two ILP methods go further, by requiring that cycles are chosen in a way that actually gives an embedding. These methods can be obtained from the first two by modifying the constraints, as we explain below.

First, let $\mathcal{S}_{\{v, k\}}$ be the set of subsets of size $k$ of the neighbourhood $X(v)$ of a vertex $v$, and for any such subset $S \in \mathcal{S}_{\{v, k\}}$, let $\mathcal{C}_{S}$ be the set of cycles in $\mathcal{C}$ that contain a 2-arc of the form $(a, v, b)$ such that $a, b \in S$.

We now alter the constraints in our first method (which does not distinguish between non-orientable and orientable embeddings), to the following:

- $x_{C} \in\{0,1\}$ for all $C \in \mathcal{C}$,

- $\sum_{C \in \mathcal{C}_{e}} x_{C}=2$ for every edge $e$ of $X$, and

- $\sum_{C \in \mathcal{C}_{S}} x_{C}<k$ for every $S \in \mathcal{S}_{\{v, k\}}$, for every $v \in V(X)$ and $2 \leq k \leq\left\lfloor\frac{\operatorname{deg}(v)}{2}\right\rfloor$.

Similarly, we alter the constraints in our second method (for finding orientable embeddings only), by considering arcs instead of edges in the second constraint. The following lemma explains why these modifications help us find minimum genus embeddings.

Lemma 5.1. In the third and fourth ILP methods presented above, a feasible region consists of the set of all embeddings and all orientable embeddings of $X$, respectively, and every feasible solution that maximises the objective function $\sum_{C \in \mathcal{C}} x_{C}$ gives a minimum genus embedding of $X$.

Proof. The constraint $\sum_{C \in \mathcal{C}_{e}} x_{C}=2$ ensures that every edge is used in the embedding exactly twice. In particular, every edge lies in two faces, and so every vertex $v$ occurs $\operatorname{deg}(v)$ times among the set of faces in a feasible solution. Next, the constraints of the form $\sum_{C \in \mathcal{C}_{S}} x_{C}<k$ ensure that the faces around each vertex can be arranged into a rotation system. For if that were not possible, then the faces around some vertex $v$ would partition into rotation sub-systems, and at least one of those sub-systems would consist of $k$ faces for some $k \leq\left\lfloor\frac{\operatorname{deg}(v)}{2}\right\rfloor$, but the relevant constraint makes that impossible.

An arbitrary embedding of $X$ is given by selection of cycles with the property that each edge occurs twice, each vertex occurs $\operatorname{deg}(v)$ times, and there is a rotation system around each vertex. Hence the feasible region consists of all possible embeddings. Moreover, replacing the constraint on edges with the corresponding constraint on arcs is exactly what's needed to reduce the feasible region to orientable embeddings. In particular, in the orientable case only one orientation can be chosen for each cycle.

Note here that it is also possible to discard the objective function, and instead calculate the feasible region after adding a further constraint of the form $\sum_{C \in \mathcal{C}} x_{C}=F$, where $F$ is the expected number of faces. Similarly, we may separate this constraint into a number of other constraints specifying the number of cycles that can bound faces of particular lengths. 


\subsection{Some applications}

Example 5.2. The Folkman graph.

This is the smallest semi-symmetric finite graph. It is bipartite of order 20, with valency, diameter and girth 4, and its automorphism group has order 3840. An upper bound on the number of faces of any embedding is $2|E| / 4=80 / 4=20$. An easy computation shows there are 30 cycles of length 4 , and 80 cycles of length 6 .

Using our independence number approach with $\mathcal{C}$ taken as the set of all 4-cycles, we find that any embedding (whether orientable or non-orientable) has at most 10 faces of length 4 . Then taking $\mathcal{C}$ as the set of all 4 -cycles and all 6 -cycles, the independence number approach does not help, because the bound it gives is too large. (A reason for this is that it can allow three cycles that are pairwise independent in the auxiliary graph $X_{\mathcal{C}}$ but cannot occur simultaneously as bounding cycles of faces of an embedding.)

On the other hand, the ILP method works very well, and tells us easily that any embedding has at most 15 faces of length up to 6 . Together these 15 faces would use up at least $(10 \cdot 4+5 \cdot 6) / 2=35$ of the 40 edges, and it then follows that the number of faces is at most 16 . But also our subgroup orbit method finds many embeddings with exactly 16 faces, which are therefore of minimum genus.

The most symmetric non-orientable embeddings of minimum genus have ten faces of length 4, five of length 6 and one of length 10, with a dihedral automorphism group of order 10 . With a suitable labelling of the vertices, one such group $S$ is generated by the elements that induce the permutations

$$
(2,3)(4,5)(7,8)(9,10)(11,19)(12,16)(14,18)(17,20)
$$

and

$$
(1,4,2,3,5)(6,9,7,8,10)(11,17,13,20,19)(12,14,15,18,16),
$$

and then the 16 faces come from the orbits of $S$ containing the 4 -cycle

$$
(1,11,6,14)
$$

the 6-cycle

$$
(1,11,9,15,10,19)
$$

and the 10-cycle

$$
(1,14,3,16,4,15,5,12,2,18) \text {. }
$$

The most symmetric orientable embeddings of minimum genus have ten faces of length 4 , four of length 6 and two of length 8 , with elementary abelian automorphism group of order 8 . With the same vertex-labelling as above, one such group $S$ is generated by the elements inducing the involutions

$$
\begin{aligned}
& (1,6)(2,7)(3,8)(4,9)(5,10) \\
& (1,2)(4,5)(6,7)(9,10)(11,12)(13,14)(16,20)(17,19)
\end{aligned}
$$

and

$$
(1,4)(2,5)(6,9)(7,10)(13,20)(14,16)(15,18)(17,19) \text {, }
$$


and then the 16 faces come from the orbits of $S$ containing the 4-cycles
$(1,11,6,14)$
$(1,18,6,19)$
$(3,14,8,16)$,

the 6-cycle

$$
(1,14,3,13,2,18)
$$

and the 8-cycle

$$
(1,19,10,12,7,17,4,11) \text {. }
$$

Also the three given generators of $S$ all reverse orientation, so this embedding is reflexible.

Accordingly, we have the following theorem.

Theorem 5.3. The minimum orientable genus of the Folkman graph is 3, attainable by a reflexible embedding with 16 faces, of which ten have length 4 , four have length 6 , and two have length 8, and with elementary abelian automorphism group of order 8. The minimum non-orientable genus of the Folkman graph is 6 , attainable by an embedding with 16 faces, in which ten have length 4 , five have length 6 , and one has length 10 , and with dihedral automorphism group of order 10.

Example 5.4. The Doyle-Holt graph.

The Doyle-Holt graph is the smallest finite graph that is half-arc-transitive, meaning that it is vertex- and edge-transitive but not arc-transitive. It was discovered independently by Doyle (and mentioned in his Harvard thesis in 1976) and Holt in 1981 (see [25]). This graph has order 27 , valency 4 , diameter 3 and girth 5 , and its automorphism group has order 54. It is also isomorphic to a spanning subgraph of the Menger graph of the dual of the Gray configuration, which we deal with in the next example.

An upper bound on the number of faces of any embedding is $\lfloor 108 / 5\rfloor=21$, and an easy computation shows there are 54 cycles of length 5 , and 63 cycles of length 6 . Our independence number method gives 27 as an upper bound on the number of faces of length 5 , but our ILP method gives an upper bound of 18. Also if an embedding has 21 faces, with $F_{\ell}$ of length greater than 5 , we have $108=2|E| \geq 5 F_{5}+6 F_{\ell}=5\left(F_{5}+F_{\ell}\right)+F_{\ell}=$ $105+F_{\ell}$, and so $F_{\ell} \leq 3$, and it follows that $\left(F_{5}, F_{\ell}\right)=(18,3)$.

Our orbit method gives such a non-orientable embedding with 21 faces, and automorphism group isomorphic to $D_{3} \times C_{3}$, of order 18 . With a suitable labelling of the vertices, this group can be generated by the elements inducing the permutations

$$
\begin{aligned}
& (1,2,3)(4,5,6)(7,8,9)(10,11,12)(13,14,15) \\
& (16,17,18)(19,20,21)(22,23,24)(25,26,27) \\
& (2,3)(5,6)(8,9)(10,21)(11,20)(12,19)(13,24) \\
& (14,23)(15,22)(16,27)(17,26)(18,25)
\end{aligned}
$$

and

$$
\begin{aligned}
& (1,4,7)(2,5,8)(3,6,9)(10,13,16)(11,14,17) \\
& (12,15,18)(19,22,25)(20,23,26)(21,24,27),
\end{aligned}
$$

and then the 21 faces of the embedding come from the orbits containing the 5 -cycle

$$
(1,13,8,19,18)
$$


and the 6-cycle

$$
(10,23,16,20,13,26) \text {. }
$$

For orientable embeddings on the other hand, the second version of our ILP method (applied to oriented cycles of length 5) shows that the number of faces of length 5 is at most 14. Similarly, the oriented version of our independence number method shows this number is at most 15. In particular, it follows that an orientable embedding cannot have 21 faces (and characteristic -6), so the total number of its faces is no more than 19 .

Using our orbit method, we found there exists an orientable embedding with 19 faces, indeed with $F_{5}=14, F_{6}=1$ and $F_{8}=4$. With the same vertex-labelling as previously, the automorphism group of this embedding is the group of order 2 generated by the involutory automorphism given for the non-orientable embedding above, and then the faces come from the seven orbits of containing the 5 -cycles
$(1,13,20,5,25)$,
$(1,18,19,8,13)$,
$(2,14,21,7,22)$,
$(2,16,20,9,14)$
$(2,22,18,6,26)$,
$(2,26,10,23,16)$,
$(4,16,23,8,19)$,

the 6-cycle

$$
(12,25,15,19,18,22)
$$

and the orbits of the 8-cycles

$$
(4,12,22,7,10,5,20,16) \quad \text { and } \quad(5,10,26,13,8,11,24,17)
$$

Also the given generator preserves the face bounded by the 6 -cycle

$$
(12,25,15,19,18,22)
$$

as well as its orientation, and so this embedding is chiral.

In particular, we have found the minimum orientable genus of the Doyle-Holt graph, thereby answering a question posed in [30] and taking it further, as follows:

Theorem 5.5. The minimum orientable genus of the Doyle-Holt graph is 5, attainable by a chiral embedding with 19 faces, of which 14 have length 5, one has length 6 , and four have length 8, and automorphism group of order 2. The minimum non-orientable genus of the Doyle-Holt graph is 8, attainable by an embedding with 21 faces, of which 18 have length 5 and three have length 6 , and automorphism group of order 18 isomorphic to $D_{3} \times C_{3}$.

Example 5.6. The dual Menger graph of the Gray configuration.

The Gray configuration is a configuration of 27 points and 27 lines, which can be realised in 3-dimensional Euclidean space via a $3 \times 3 \times 3$ grid, with the lines as pairwise intersections of 9 planes, partitioned into three triples, each being parallel to one of the three planes with equations $x=0, y=0$ and $z=0$.

The Gray graph is the Levi graph (or incidence graph) of this configuration, namely the bipartite graph whose vertices are the points and lines and whose edges represent incidence (between points and lines). Also the Menger graph of the Gray configuration, which indicates collinearity of points, is isomorphic to the Cartesian product $C_{3} \square C_{3} \square C_{3}$. On the other hand, the Menger graph of the dual of the Gray configuration, which indicates 
copunctuality of lines, is another graph of order 27 , valency 6 , diameter 3 and girth 3 , with automorphism group of order 1296. It was studied in some detail in [30].

Let $X$ be this dual Menger graph. As with $C_{3} \square C_{3} \square C_{3}$, an upper bound on the number of faces of an embedding of $X$ is $162 / 3=54$, but that bound is far from sharp. By inspection (or an easy application of our ILP method) there can be at most 27 faces of length 3, and from this it follows that the number of faces is bounded above by 47 . Better still, if we take $\mathcal{C}_{j}$ as the set of all cycles of length $j$ for $j \in\{3,4\}$, and then $\mathcal{C}=\mathcal{C}_{3} \cup \mathcal{C}_{4}$, our ILP method gives a maximum value for the objective function $2 \sum_{C \in \mathcal{C}_{3}}+\sum_{C \in \mathcal{C}_{4}}$ as 66 , and so $2 F_{3}+F_{4} \leq 66$. Hence if $F_{\ell}$ denotes the number of faces of length greater than 4 , we have

$$
162=2|E| \geq 3 F_{3}+4 F_{4}+5 F_{\ell}=5\left(F_{3}+F_{4}+F_{\ell}\right)-\left(2 F_{3}+F_{4}\right) \geq 5|F|-66,
$$

which gives $|F| \leq\lfloor(162+66) / 5\rfloor=45$.

Our subgroup orbit method provides a non-orientable embedding with 45 faces, such that $\left(F_{3}, F_{4}\right)=(18,27)$. Its automorphism group has order 108 , and is isomorphic to a semi-direct product of the non-abelian group of order 27 and exponent 3 by the Klein 4-group $V_{4}$. With a suitable labelling of the vertices, this group can be generated by the elements inducing the permutations

$$
\begin{aligned}
& (1,25)(2,11)(4,22)(5,24)(6,9)(7,27)(8,17)(10,26)(12,20) \\
& (13,21)(14,15)(16,19)
\end{aligned}
$$

and

$$
(1,2,15)(3,14,4,5,9,10)(6,19,7,11,18,8)(12,13,24,26,17,16)
$$

$(20,23,21,27,25,22)$,

and then the $18+27=45$ faces of the embedding come from the orbits of the 3 -cycle $(1,4,8)$ and 4 -cycle $(1,2,3,7)$. The characteristic of this embedding is

$$
\chi=27-81+45=-9 .
$$

Next, for orientable embeddings, there can be at most 44 faces (in order to have even characteristic), and our orbit method produces one with $\left(F_{3}, F_{4}, F_{6}\right)=(26,12,6)$. With the same vertex-labelling as previously, the automorphism group of this one is cyclic of order 6 , generated by the second of the two automorphisms given for the non-orientable embedding above. The 44 faces come from the eight orbits containing the five 3 -cycles

$$
(1,4,8), \quad(3,8,17), \quad(3,27,7), \quad(12,23,20), \quad(12,24,17),
$$

the two 4-cycles

$$
(1,8,3,2), \quad(3,17,25,27),
$$

and the single 6 -cycle

$$
(4,18,26,16,20,8) \text {. }
$$

Also the given generator reverses orientation, and so this embedding is reflexible.

In particular, we have found the minimum orientable genus of this graph, thereby answering a question posed in [30], and taking it further, as follows: 
Theorem 5.7. The minimum orientable genus of the dual Menger graph of the Gray configuration is 6 , attainable by a reflexible embedding with 44 faces, of which 26 have length 3 , and 12 have length 4, and 6 have length 6 , with a cyclic automorphism group of order 6 . The minimum non-orientable genus of the same graph is 11, attainable by an embedding with 45 faces, of which 18 have length 3, and 27 have length 4, and automorphism group of order 108 isomorphic to a semi-direct product of the non-abelian group of order 27 and exponent 3 by the Klein 4-group.

Also in [30] it was noted that if $H$ and $D$ are the Doyle-Holt graph and the Menger graph of the dual Gray configuration, respectively, then $4 \leq \gamma(H) \leq \gamma(D) \leq 7$. We have now shown that $\gamma(H)=5$ and $\gamma(D)=6$, so that in fact $4<\gamma(H)<\gamma(D)<7$.

Moreover, it was shown in [30, Proposition 1] that if $L$ is the Levi graph and $M$ is the Menger graph of any $\left(v_{3}\right)$ configuration, then $\gamma(M) \leq \gamma(L)$, and near the end of [30] the authors asked about finding such a configuration for which that inequality in strict. Our work provides an answer to this question as well, because the Levi graph of the dual of the Gray configuration is the Gray graph (giving $\gamma(L)=7$ ), while its Menger graph is the above graph $D$, with $\gamma(D)=6<7$. Hence we can strengthen Proposition 1 of [30] to the following:

Theorem 5.8. If $L$ is the Levi graph and $M$ is the Menger graph of any $\left(v_{3}\right)$ configuration, then $\gamma(M) \leq \gamma(L)$, and this inequality is strict for the dual of the Gray $\left(27_{3}\right)$ configuration.

Example 5.9. The second smallest semi-symmetric cubic graph.

This graph is also the smallest 'Iofinova-Ivanov graph', constructed in [26], and so we will call it $I I_{1}$. It was the most challenging of all the examples we considered in this work. It is a bipartite graph of order 110, diameter 7 and girth 10, with automorphism group of order 1320, isomorphic to $\operatorname{PGL}(2,11)$; see [8] for more details.

The naive upper bound on the number of faces of any embedding of $I I_{1}$ is $330 / 10=33$, but again this is not sharp. Using our ILP approach on cycles of length 10 and 12, it can be shown that $2 F_{10}+F_{12}$ is at most 48 , and hence if $F_{\ell}$ is the number of faces of length greater than 12 , we have

$$
\begin{aligned}
330=2|E| & \geq 10 F_{10}+12 F_{12}+14 F_{\ell} \\
& =14\left(F_{10}+F_{12}+F_{\ell}\right)-2\left(2 F_{10}+F_{12}\right) \\
& \geq 14|F|-96,
\end{aligned}
$$

and therefore $|F| \leq\lfloor 426 / 14\rfloor=30$.

Now this improved upper bound is sharp, because our orbit method produces a nonorientable embedding with exactly 30 faces, and $F_{10}=F_{12}=15$. With a suitable labelling of vertices, the automorphism group $S$ of this embedding is cyclic of order 3 , generated by the automorphism inducing the permutation

$$
\begin{aligned}
& (1,33,46)(2,52,18)(3,10,24)(4,17,13)(5,15,41)(6,53,32)(7,31,27) \\
& (8,26,42)(9,47,22)(11,38,51)(12,44,16)(14,34,28)(19,36,43)(20,40,54) \\
& (21,45,30)(23,48,35)(25,39,29)(49,55,50)(56,91,84)(57,74,93) \\
& (58,80,103)(59,110,65)(60,101,94)(62,86,77)(64,82,92)(66,102,104) \\
& (67,95,97)(68,72,99)(69,73,105)(70,79,78)(71,90,100)(75,85,106) \\
& (76,108,88)(83,87,107)(89,96,98),
\end{aligned}
$$


and then the faces of the embedding come from the $S$-orbits of the five 10 -cycles

$$
\begin{aligned}
& (1,56,2,59,6,66,19,68,8,57), \\
& (2,56,4,62,11,83,29,78,14,60), \\
& (3,64,23,96,45,73,26,74,10,61), \\
& (5,70,25,76,12,82,48,100,32,65), \\
& (6,71,20,88,29,83,43,104,37,66),
\end{aligned}
$$

and the five 12-cycles

$$
\begin{aligned}
& (1,56,4,63,13,77,16,88,20,67,7,58), \\
& (1,57,3,64,16,77,51,106,34,70,5,58), \\
& (2,59,15,80,31,99,42,105,47,81,9,60), \\
& (7,67,30,98,49,109,55,106,51,107,36,72) \quad \text { and } \\
& (9,60,14,85,49,98,48,100,54,97,45,73) .
\end{aligned}
$$

The characteristic is $\chi=110-165+30=-25$.

Our subgroup orbit method also produces an orientable embedding with 27 faces, indeed with $\left(F_{10}, F_{12}, F_{14}\right)=(6,12,9)$, from the same cyclic subgroup $S$ of order 3 . In particular, this embedding is chiral, because $S$ has odd order. Its faces come from the nine $S$-orbits containing the two 10-cycles

$$
\begin{aligned}
& (1,56,2,60,9,73,26,72,7,58), \\
& (11,75,50,96,23,64,16,88,29,83),
\end{aligned}
$$

the four 12-cycles

$$
\begin{aligned}
& (1,57,8,69,21,95,40,76,12,62,4,56), \\
& (1,58,5,70,34,106,51,77,16,64,3,57), \\
& (2,56,4,63,17,86,38,87,19,66,6,59), \\
& (5,65,32,100,54,97,27,68,19,87,25,70),
\end{aligned}
$$

and the three 14-cycles

$$
\begin{aligned}
& (2,59,15,80,31,95,21,89,55,109,49,85,14,60), \\
& (3,64,23,90,53,102,37,104,43,99,42,93,24,61) \quad \text { and } \\
& (9,60,14,78,29,88,20,71,35,89,21,69,22,81) .
\end{aligned}
$$

In turns out that this is an orientable embedding of minimum genus, because there exist none with 29 faces (and characteristic $110-165+29=-26$ ). We were not able to prove that by using the standard forms of our independence number and ILP methods, because the size and girth of the graph create too many cycles for consideration as face boundaries. But we were still able to prove it by a slightly different approach, using restricted forms of those methods, and we now give a brief outline of the proof.

Assume there exists an orientable embedding with 29 faces, and again let $F_{k}$ denote the number of faces of length $k$. Then an easy computation of weighted sums shows there 
are 435 possibilities for the sequence $\left(F_{10}, F_{12}, F_{14}, F_{16}, F_{18}, \ldots\right)$, with the integer $F_{10}$ ranging from 9 to 23 . These possibilities can be dealt with in groups or individually, with the aim of eliminating all of them.

For example, suppose $F_{12} \geq 1$, and let $\mathcal{C}$ be the set of all directed 10 - and 12-cycles in $I I_{1}$. Now let $C$ and $D$ be any directed 10-cycle and 12-cycle that are independent in the auxiliary graph $X_{\mathcal{C}}$, and let $\mathcal{C}_{(C D)}$ be the subset of $\mathcal{C}$ consisting of all directed 10-cycles that are independent of both $C$ and $D$ in $X_{\mathcal{C}}$. We then compute the independence number of the auxiliary graph $X_{\mathcal{C}_{(C D)}}$ for all such pairs $(C, D)$, up to equivalence in the automorphism group of the graph. This computation shows that the maximum of these independence numbers is 21 , from which it follows that $F_{10} \leq 22$ when $F_{12} \geq 1$. This eliminates 70 possibilities for the sequence $\left(F_{10}, F_{12}, F_{14}, F_{16}, F_{18}, \ldots\right)$.

Similarly, another 57 possibilities can be eliminated in the case where $F_{12} \geq 2$, for in that case the corresponding independence number computation shows that $F_{10} \leq 21$, and another 71 can be eliminated when $F_{12} \geq 4$, for in that case $F_{10} \leq 19$, and then another 30 when $F_{12} \geq 5$, and another 42 when $F_{14} \geq 1$ (with no assumption on $F_{12}$ ). Other cases that help eliminate possibilities include those where both $F_{14} \geq 1$ and $F_{16} \geq 1$, and so on.

This kind of approach reduced the problem to just six possibilities, namely those for which

$$
\begin{aligned}
\left(F_{10}, F_{12}, F_{14}, F_{16}, F_{18}\right)=(18,6,1,4,0),(18,6,2,2,1),(17,7,2,3,0), \\
(19,4,2,4,0),(16,6,7,0,0) \text { and }(20,3,1,5,0),
\end{aligned}
$$

all but one of which could be eliminated by similar means. For some of them, we used the ILP method in place of the independence method, when the independence method gave too large a number.

The trickiest case was the last of the above six possibilities, namely the one where $\left(F_{10}, F_{12}, F_{14}, F_{16}\right)=(20,3,1,5)$. For this, we took $\mathcal{C}$ be the set of all directed 10-, 12-, 14- and 16-cycles in $I I_{1}$, and ran through all possibilities for a quintuple $Q$ of independent vertices in the auxiliary graph $X_{\mathcal{C}}$, consisting of three 12 -cycles, one 14 -cycle and one 16 cycle, and for each one, determined the maximum size of a set $T$ of 10-cycles for which $Q \cup T$ is an independent set in $X_{\mathcal{C}}$. The maximum size found was 18 , indicating that if $F_{12}=3$ and $F_{14}=1$ and $F_{16} \geq 1$, then $F_{10} \leq 18$. In particular, this shows that $\left(F_{10}, F_{12}, F_{14}, F_{16}\right)$ cannot be $(20,3,1,5)$.

Hence the number of faces of an orientable embedding of $I I_{1}$ cannot be 29, and we have an answer to the penultimate open question in [30]. We state this the first part of the following, to complete the paper:

Theorem 5.10. The minimum orientable genus of the second smallest semi-symmetric cubic graph $I I_{1}$ is 15, attainable by a chiral embedding with 27 faces, of which six have length 10, twelve have length 12 , and nine have length 14 , and with cyclic automorphism group of order 3. The minimum non-orientable genus of the same graph is 27 , attainable by an embedding with 30 faces, of which 15 have length 10, and 15 have length 12, and with cyclic automorphism group of order 3 (acting in the same way on the graph).

\section{Final remarks}

In this paper we have presented four new methods that are helpful for determining the minimum genus of embeddings of a graph on a surface. Also we have shown in some detail how counting arguments can be of great use in solving this kind of problem. 
Our first one (the subgroup orbit method) considers possibilities for a group of automorphisms of the embedding, of suitable order, thereby reducing the search space. A suitable set of candidate faces is formed from closed walks of appropriate lengths in the graph, and then the faces are chosen from orbits of the chosen group on those walks.

The second one (the independence number method) uses the independence number of an auxiliary graph to bound the maximum number of faces of given lengths. This method can be very useful when taken in combination with other approaches. In particular, it can be used to determine that no embedding of a given graph can have certain numbers of faces of given lengths, even when counting arguments do not help.

Our third and the fourth methods translate the problem of choosing faces from a set of candidate closed walks into a linear programming problem. The third method can help find upper bounds on the number of faces of particular lengths that can be used in an embedding, while the fourth method aims to find an actual embedding of the graph with minimum genus. This is based on an approach that translates the conditions for a set of closed walks of the graph to give an embedding into to a set of linear constraints and an objective function for minimising the genus.

All of these methods use a set of closed walks of the graph (for bounding candidate faces). Since the set of all closed walks of up to given length in the graph can be enormous, it is best to use these methods in combination with counting arguments, to limit (or rule out) the lengths of candidate faces. This is often easily done by hand, but can also be automated. Also our methods can be used more generally to find embeddings of a graph with given face lengths, and are not necessarily restricted to finding minimum genus embeddings.

Our linear programming method for calculating an explicit embedding provides a relatively fast way of finding a minimum genus embedding of a graph, without considering symmetries. It is possible to combine this with prescription of symmetries (indeed, this is a standard trick in linear programming), but the obvious way of doing that involves reducing the problem to finding an embedding of a quotient (voltage) graph. Also it can be difficult to prescribe the lengths of faces of the latter embedding, as seen in Subsection 3.4). The subgroup orbit method is better suited in many cases, because it provides complete control over the lengths of the faces of the cover. Indeed, this has proven very successful in the cases of vertex-, edge- or arc-transitive graphs.

Finally, we have demonstrated how to use these methods with several examples, in which we determined the minimum genus of embeddings of several well-known interesting graphs, either for the first time, or in a different (and sometimes better) way than achieved previously. We have stored details of these minimum genus embeddings at the $A M C$ website associated with this article.

\section{References}

[1] S. Beyer, M. Chimani, I. Hedtke and M. Kotrbčík, A practical method for the minimum genus of a graph: models and experiments, in: A. V. Goldberg and A. S. Kulikov (eds.), Experimental Algorithms, Springer, Cham, volume 9685 of Lecture Notes in Computer Science, pp. 75-88, 2016, doi:10.1007/978-3-319-38851-9_6, proceedings of the 15th International Symposium (SEA 2016) held in St. Petersburg, June 5-8, 2016.

[2] W. Bosma, J. Cannon and C. Playoust, The Magma algebra system I: The user language, J. Symbolic Comput. 24 (1997), 235-265, doi:10.1006/jsco.1996.0125.

[3] I. Z. Bouwer, On edge but not vertex transitive regular graphs, J. Comb. Theory Ser. B 12 (1972), 32-40, doi:10.1016/0095-8956(72)90030-5. 
[4] M. G. Brin and C. C. Squier, On the genus of $\mathbb{Z}_{3} \times \mathbb{Z}_{3} \times \mathbb{Z}_{3}$, European J. Combin. 9 (1988), 431-443, doi:10.1016/s0195-6698(88)80002-7.

[5] A. E. Brouwer, I. J. Dejter and C. Thomassen, Highly symmetric subgraphs of hypercubes, J. Algebraic Combin. 2 (1993), 25-29, doi:10.1023/a:1022472513494.

[6] M. Chimani, Computing Crossing Numbers, Ph.D. thesis, Technischen Universität Dortmund, Dortmund, 2008, http://1s11-www.cs.tu-dortmund.de/people/chimani/ files/diss.pdf.

[7] M. Conder, A. Malnič, D. Marušič, T. Pisanski and P. Potočnik, The edge-transitive but not vertex-transitive cubic graph on 112 vertices, J. Graph Theory 50 (2005), 25-42, doi:10.1002/ jgt.20089.

[8] M. Conder, A. Malnič, D. Marušič and P. Potočnik, A census of semisymmetric cubic graphs on up to 768 vertices, J. Algebraic Combin. 23 (2006), 255-294, doi:10.1007/s10801-006-7397-3.

[9] M. D. E. Conder, J. Širáň and T. W. Tucker, The genera, reflexibility and simplicity of regular maps, J. Eur. Math. Soc. 12 (2010), 343-364, doi:10.4171/jems/200.

[10] H. S. M. Coxeter, Self-dual configurations and regular graphs, Bull. Amer. Math. Soc. 56 (1950), 413-455, doi:10.1090/s0002-9904-1950-09407-5.

[11] H. S. M. Coxeter and W. O. J. Moser, Generators and Relations for Discrete Groups, volume 14 of Ergebnisse der Mathematik und ihrer Grenzgebiete, Springer, Berlin, 4th edition, 1980.

[12] R. A. Duke, The genus, regional number, and Betti number of a graph, Canad. J. Math. 18 (1966), 817-822, doi:10.4153/cjm-1966-081-6.

[13] W. Dyck, Beiträge zur Analysis situs, Math. Ann. 32 (1888), 457-512, doi:10.1007/ bf01443580.

[14] I. S. Filotti, G. L. Miller and J. Reif, On determining the genus of a graph in $O\left(v^{O(g)}\right)$ steps, in: M. J. Fischer, R. A. DeMillo, N. A. Lynch, W. A. Burkhard and A. V. Aho (eds.), STOC'79: Proceedings of the 11th Annual ACM Symposium on Theory of Computing, ACM Press, New York, 1979 pp. 27-37, doi:10.1145/800135.804395, held in Atlanta, Georgia, April 30 - May 02, 1979.

[15] G. Gévay and T. Pisanski, Kronecker covers, $V$-construction, unit-distance graphs and isometric point-circle configurations, Ars Math. Contemp. 7 (2014), 317-336, doi:10.26493/ 1855-3974.359.8eb.

[16] M. J. Grannell and T. S. Griggs, Designs and topology, in: A. Hilton and J. Talbot (eds.), Surveys in Combinatorics 2007, Cambridge University Press, Cambridge, volume 346 of London Mathematical Society Lecture Note Series, pp. 121-174, 2007, doi:10.1017/ cbo9780511666209.006, papers from the 21st Biennial British Combinatorial Conference held in Reading, July 8 - 13, 2007.

[17] M. J. Grannell and T. S. Griggs, Embedding and designs, in: L. W. Beineke and R. J. Wilson (eds.), Topics in Topological Graph Theory, Cambridge University Press, Cambridge, volume 128 of Encyclopedia of Mathematics and its Applications, pp. 268-288, 2009, doi:10.1017/ cbo9781139087223.016.

[18] J. L. Gross and T. W. Tucker, Generating all graph coverings by permutation voltage assignments, Discrete Math. 18 (1977), 273-283, doi:10.1016/0012-365x(77)90131-5.

[19] J. L. Gross and T. W. Tucker, Topological Graph Theory, Wiley-Interscience Series in Discrete Mathematics and Optimization, John Wiley \& Sons, New York, 1987.

[20] B. Grünbaum, Configurations of Points and Lines, volume 103 of Graduate Studies in Mathematics, American Mathematical Society, Providence, Rhode Island, 2009, doi:10.1090/gsm/ 103. 
[21] P. R. Hafner, The Hoffman-Singleton graph and its automorphisms, J. Algebraic Combin. 18 (2003), 7-12, doi:10.1023/a:1025136524481.

[22] L. Heffter, Ueber das Problem der Nachbargebiete, Math. Ann. 38 (1891), 477-508, doi:10. 1007/bf01203357.

[23] L. Heffter, Ueber Tripelsysteme, Math. Ann. 49 (1897), 101-112, doi:10.1007/bf01445363.

[24] D. Hilbert and S. Cohn-Vossen, Geometry and the Imagination, Chelsea Publishing Company, New York, New York, 1952, translated by P. Neményi.

[25] D. F. Holt, A graph which is edge transitive but not arc transitive, J. Graph Theory 5 (1981), 201-204, doi:10.1002/jgt.3190050210.

[26] M. E. Iofinova and A. A. Ivanov, Biprimitive cubic graphs, in: I. A. Faradžev, A. A. Ivanov, M. H. Klin and A. J. Woldar (eds.), Investigations in the Algebraic Theory of Combinatorial Objects, Kluwer Academic Publishers Group, Dordrecht, volume 84 of Mathematics and its Applications (Soviet Series), pp. 459-472, 1994, doi:10.1007/978-94-017-1972-8_16.

[27] B. Jackson, T. D. Parsons and T. Pisanski, A duality theorem for graph embeddings, J. Graph Theory 5 (1981), 55-77, doi:10.1002/jgt.3190050104.

[28] P. Kaski and P. R. J. Östergård, Classification Algorithms for Codes and Designs, volume 15 of Algorithms and Computation in Mathematics, Springer-Verlag, Berlin, 2006, doi:10.1007/ 3-540-28991-7.

[29] A. Malnič, R. Nedela and M. Škoviera, Lifting graph automorphisms by voltage assignments, European J. Combin. 21 (2000), 927-947, doi:10.1006/eujc.2000.0390.

[30] D. Marušič, T. Pisanski and S. Wilson, The genus of the GRAY graph is 7, European J. Combin. 26 (2005), 377-385, doi:10.1016/j.ejc.2004.01.015.

[31] B. Mohar, A linear time algorithm for embedding graphs in an arbitrary surface, SIAM J. Discrete Math. 12 (1999), 6-26, doi:10.1137/s089548019529248x.

[32] B. Mohar, T. Pisanski, M. Škoviera and A. White, The Cartesian product of three triangles can be embedded into a surface of genus 7, Discrete Math. 56 (1985), 87-89, doi:10.1016/ 0012-365x(85)90197-9.

[33] P. Mutzel and R. Weiskircher, Optimizing over all combinatorial embeddings of a planar graph (Extended abstract), in: G. Cornuéjols, R. E. Burkard and G. J. Woeginger (eds.), Integer Programming and Combinatorial Optimization, Springer, Berlin, volume 1610 of Lecture Notes in Computer Science, 1999 pp. 361-376, doi:10.1007/3-540-48777-8_27, proceedings of the 7th International Conference (IPCO VII) held in Graz, June 9 - 11, 1999.

[34] W. Myrvold and W. Kocay, Errors in graph embedding algorithms, J. Comput. System Sci. 77 (2011), 430-438, doi:10.1016/j.jcss.2010.06.002.

[35] T. D. Parsons, G. Pica, T. Pisanski and A. G. S. Ventre, Orientably simple graphs, Math. Slovaca 37 (1987), 391-394.

[36] T. D. Parsons, T. Pisanski and B. Jackson, Dual imbeddings and wrapped quasi-coverings of graphs, Discrete Math. 31 (1980), 43-52, doi:10.1016/0012-365x(80)90170-3.

[37] T. Pisanski and B. Servatius, Configurations from a Graphical Viewpoint, Birkhäuser Advanced Texts Basler Lehrbücher, Birkhäuser, New York, 2013, doi:10.1007/978-0-8176-8364-1.

[38] G. Ringel, Bestimmung der Maximalzahl der Nachbargebiete auf nichtorientierbaren Flächen, Math. Ann. 127 (1954), 181-214, doi:10.1007/bf01361120.

[39] G. Ringel, Das Geschlecht des vollständigen paaren Graphen, Abh. Math. Sem. Univ. Hamburg 28 (1965), 139-150, doi:10.1007/bf02993245. 
[40] G. Ringel and J. W. T. Youngs, Solution of the Heawood map-coloring problem, Proc. Nat. Acad. Sci. USA 60 (1968), 438-445, doi:10.1073/pnas.60.2.438.

[41] G. N. Robertson, Graphs Minimal under Girth, Valency and Connectivity Constraints, Ph.D. thesis, University of Waterloo, Waterloo, Ontario, Canada, 1969, https://search. proquest.com/docview/302450139.

[42] N. Robertson and P. D. Seymour, Graph minors. VIII. A Kuratowski theorem for general surfaces, J. Comb. Theory Ser. B 48 (1990), 255-288, doi:10.1016/0095-8956(90)90121-f.

[43] M. Škoviera and R. Nedela, The maximum genus of vertex-transitive graphs, Discrete Math. 78 (1989), 179-186, doi:10.1016/0012-365x(89)90175-1.

[44] S. Stahl, The embedding of a graph—a survey, J. Graph Theory 2 (1978), 275-298, doi:10. 1002/jgt.3190020402.

[45] W. A. Stein et al., Sage Mathematics Software (Version 6.0), The Sage Development Team, 2013, http: / /www. sagemath.org.

[46] K. Stokes and M. Izquierdo, Geometric point-circle pentagonal geometries from Moore graphs, Ars Math. Contemp. 11 (2016), 215-229, doi:10.26493/1855-3974.787.925.

[47] C. Thomassen, The graph genus problem is NP-complete, J. Algorithms 10 (1989), 568-576, doi:10.1016/0196-6774(89)90006-0.

[48] H. Van Maldeghem, Ten exceptional geometries from trivalent distance regular graphs, Ann. Comb. 6 (2002), 209-228, doi:10.1007/p100012587.

[49] A. T. White, Graphs, Groups and Surfaces, volume 8 of North-Holland Mathematics Studies, North-Holland, Amsterdam, 2nd edition, 1984.

[50] A. T. White, Graphs of Groups on Surfaces. Interactions and Models, volume 188 of NorthHolland Mathematics Studies, North-Holland, Amsterdam, 2001.

[51] N. H. Xuong, How to determine the maximum genus of a graph, J. Comb. Theory Ser. B 26 (1979), 217-225, doi:10.1016/0095-8956(79)90058-3.

[52] J. W. T. Youngs, Minimal imbeddings and the genus of a graph, J. Math. Mech. 12 (1963), 303-315. 